DESY 00-187

ISSN 0418-9833

December 2000

\title{
Measurement of Neutral and Charged Current Cross Sections in Electron-Proton Collisions at High $Q^{2}$
}

\author{
H1 Collaboration
}

\begin{abstract}
The inclusive $e^{-} p$ single and double differential cross sections for neutral and charged current processes are measured with the $\mathrm{H} 1$ detector at HERA, in the range of four-momentum transfer squared $Q^{2}$ between 150 and $30000 \mathrm{GeV}^{2}$, and Bjorken $x$ between 0.002 and 0.65. The data were taken in 1998 and 1999 with a centre-of-mass energy of $320 \mathrm{GeV}$ and correspond to an integrated luminosity of $16.4 \mathrm{pb}^{-1}$. The data are compared with recent measurements of the inclusive neutral and charged current $e^{+} p$ cross sections. For $Q^{2}>1000 \mathrm{GeV}^{2}$ clear evidence is observed for an asymmetry between $e^{+} p$ and $e^{-} p$ neutral current scattering and the generalised structure function $x \tilde{F}_{3}$ is extracted for the first time at HERA. A fit to the charged current data is used to extract a value for the $W$ boson propagator mass. The data are found to be in good agreement with Standard Model predictions.
\end{abstract}

To be submitted to Eur. Phys. J. C 
C. Adloff ${ }^{33}$, V. Andreev ${ }^{24}$, B. Andrieu ${ }^{27}$, T. Anthonis ${ }^{4}$, V. Arkadov ${ }^{35}$, A. Astvatsatourov ${ }^{35}$, I. Ayyaz $^{28}$, A. Babaev ${ }^{23}$, J. Bähr ${ }^{35}$, P. Baranov ${ }^{24}$, E. Barrelet ${ }^{28}$, W. Bartel ${ }^{10}$, U. Bassler ${ }^{28}$, P. Bate ${ }^{21}$, A. Beglarian ${ }^{34}$, O. Behnke ${ }^{13}$, C. Beier ${ }^{14}$, A. Belousov ${ }^{24}$, T. Benisch ${ }^{10}$, Ch. Berger $^{1}$, G. Bernardi ${ }^{28}$, T. Berndt ${ }^{14}$, J.C. Bizot ${ }^{26}$, V. Boudry ${ }^{27}$, W. Braunschweig ${ }^{1}$, V. Brisson ${ }^{26}$, H.B. Bröker ${ }^{2}$, D.P. Brown ${ }^{11}$, W. Brückner ${ }^{12}$, P. Bruel ${ }^{27}$, D. Bruncko ${ }^{16}$, J. Bürger ${ }^{10}$, F.W. Büsser ${ }^{11}$, A. Bunyatyan ${ }^{12,34}$, H. Burkhardt ${ }^{14}$, A. Burrage ${ }^{18}$, G. Buschhorn ${ }^{25}$, A.J. Campbell ${ }^{10}$, J. Cao ${ }^{26}$, T. Carli ${ }^{25}$, S. Caron ${ }^{1}$, E. Chabert ${ }^{22}$, D. Clarke ${ }^{5}$, B. Clerbaux ${ }^{4}$, C. Collard ${ }^{4}$, J.G. Contreras ${ }^{7,41}$, Y.R. Coppens ${ }^{3}$, J.A. Coughlan ${ }^{5}$, M.-C. Cousinou ${ }^{22}$, B.E. Cox ${ }^{21}$, G. Cozzika ${ }^{9}$, J. Cvach ${ }^{29}$, J.B. Dainton ${ }^{18}$, W.D. Dau ${ }^{15}$, K. Daum ${ }^{33,39}$, M. Davidsson ${ }^{20}$, B. Delcourt ${ }^{26}$, N. Delerue ${ }^{22}$, R. Demirchyan ${ }^{34}$, A. De Roeck ${ }^{10,43}$, E.A. De Wolf ${ }^{4}$, C. Diaconu ${ }^{22}$, P. Dixon ${ }^{19}$, V. Dodonov ${ }^{12}$, J.D. Dowell ${ }^{3}$,

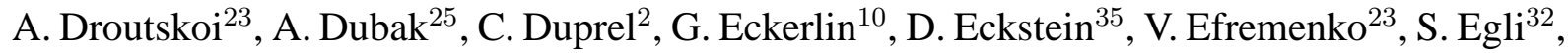
R. Eichler ${ }^{36}$, F. Eisele ${ }^{13}$, E. Eisenhandler ${ }^{19}$, M. Ellerbrock ${ }^{13}$, E. Elsen ${ }^{10}$, M. Erdmann ${ }^{10,40, e}$, W. Erdmann ${ }^{36}$, P.J.W. Faulkner ${ }^{3}$, L. Favart ${ }^{4}$, A. Fedotov ${ }^{23}$, R. Felst ${ }^{10}$, J. Ferencei ${ }^{10}$, S. Ferron $^{27}$, M. Fleischer ${ }^{10}$, Y.H. Fleming ${ }^{3}$, G. Flügge ${ }^{2}$, A. Fomenko ${ }^{24}$, I. Foresti ${ }^{37}$, J. Formánek ${ }^{30}$, J.M. Foster ${ }^{21}$, G. Franke ${ }^{10}$, E. Gabathuler ${ }^{18}$, K. Gabathuler ${ }^{32}$, J. Garvey ${ }^{3}$, J. Gassner ${ }^{32}$, J. Gayler ${ }^{10}$, R. Gerhards ${ }^{10}$, S. Ghazaryan ${ }^{34}$, L. Goerlich ${ }^{6}$, N. Gogitidze ${ }^{24}$, M. Goldberg ${ }^{28}$, C. Goodwin ${ }^{3}$, C. Grab ${ }^{36}$, H. Grässler ${ }^{2}$, T. Greenshaw ${ }^{18}$, G. Grindhammer ${ }^{25}$, T. Hadig ${ }^{13}$, D. Haidt ${ }^{10}$, L. Hajduk ${ }^{6}$, W.J. Haynes ${ }^{5}$, B. Heinemann ${ }^{18}$, G. Heinzelmann ${ }^{11}$, R.C.W. Henderson ${ }^{17}$, S. Hengstmann ${ }^{37}$, H. Henschel ${ }^{35}$, R. Heremans ${ }^{4}$, G. Herrera ${ }^{7,41}$, I. Herynek ${ }^{29}$, M. Hildebrandt ${ }^{37}$, M. Hilgers ${ }^{36}$, K.H. Hiller ${ }^{35}$, J. Hladký ${ }^{29}$, P. Höting ${ }^{2}$, D. Hoffmann ${ }^{10}$, R. Horisberger ${ }^{32}$, S. Hurling ${ }^{10}$, M. Ibbotson ${ }^{21}$, Ç. İşsever ${ }^{7}$, M. Jacquet ${ }^{26}$, M. Jaffre ${ }^{26}$, L. Janauschek ${ }^{25}$, D.M. Jansen ${ }^{12}$, X. Janssen ${ }^{4}$, V. Jemanov ${ }^{11}$, L. Jönsson ${ }^{20}$, D.P. Johnson ${ }^{4}$, M.A.S. Jones ${ }^{18}$, H. Jung ${ }^{10}$, H.K. Kästli ${ }^{36}$, D. Kant ${ }^{19}$, M. Kapichine ${ }^{8}$, M. Karlsson ${ }^{20}$, O. Karschnick ${ }^{11}$, F. Keil ${ }^{14}$, N. Keller ${ }^{37}$, J. Kennedy ${ }^{18}$, I.R. Kenyon ${ }^{3}$, S. Kermiche ${ }^{22}$, C. Kiesling ${ }^{25}$, P. Kjellberg ${ }^{20}$, M. Klein ${ }^{35}$, C. Kleinwort ${ }^{10}$, G. Knies ${ }^{10}$, B. Koblitz ${ }^{25}$, S.D. Kolya ${ }^{21}$, V. Korbel ${ }^{10}$, P. Kostka ${ }^{35}$, S.K. Kotelnikov ${ }^{24}$, R. Koutouev ${ }^{12}$, A. Koutov ${ }^{8}$, M.W. Krasny ${ }^{28}$, H. Krehbiel ${ }^{10}$, J. Kroseberg ${ }^{37}$, K. Krüger ${ }^{10}$, A. Küpper ${ }^{33}$, T. Kuhr ${ }^{11}$, T. Kurča ${ }^{35,16}$, R. Lahmann ${ }^{10}$, D. Lamb ${ }^{3}$, M.P.J. Landon ${ }^{19}$, W. Lange ${ }^{35}$, T. Laštovička ${ }^{30}$, P. Laycock ${ }^{18}$, E. Lebailly ${ }^{26}$, A. Lebedev ${ }^{24}$, B. Leißner ${ }^{1}$, R. Lemrani ${ }^{10}$, V. Lendermann ${ }^{7}$, S. Levonian ${ }^{10}$, M. Lindstroem ${ }^{20}$, B. List ${ }^{36}$, E. Lobodzinska ${ }^{10,6}$, B. Lobodzinski ${ }^{6,10}$, A. Loginov ${ }^{23}$, N. Loktionova ${ }^{24}$, V. Lubimov ${ }^{23}$, S. Lüders ${ }^{36}$, D. Lüke ${ }^{7,10}$, L. Lytkin ${ }^{12}$, N. Magnussen ${ }^{33}$, H. Mahlke-Krüger ${ }^{10}$, N. Malden ${ }^{21}$, E. Malinovski ${ }^{24}$, I. Malinovski ${ }^{24}$, R. Maraček ${ }^{25}$, P. Marage ${ }^{4}$, J. Marks ${ }^{13}$, R. Marshall ${ }^{21}$, H.U. Martyn ${ }^{1}$, J. Martyniak ${ }^{6}$, S.J. Maxfield ${ }^{18}$, A. Mehta ${ }^{18}$, K. Meier ${ }^{14}$, P. Merkel ${ }^{10}$, A.B. Meyer ${ }^{11}$, H. Meyer ${ }^{33}$, J. Meyer ${ }^{10}$, P.-O. Meyer ${ }^{2}$, S. Mikocki ${ }^{6}$, D. Milstead ${ }^{18}$, T. Mkrtchyan ${ }^{34}$, R. Mohr ${ }^{25}$, S. Mohrdieck ${ }^{11}$, M.N. Mondragon ${ }^{7}$, F. Moreau ${ }^{27}$, A. Morozov ${ }^{8}$, J.V. Morris ${ }^{5}$, K. Müller ${ }^{37}$, P. Murín ${ }^{16,42}$, V. Nagovizin ${ }^{23}$, B. Naroska ${ }^{11}$, J. Naumann ${ }^{7}$, Th. Naumann ${ }^{35}$, G. Nellen ${ }^{25}$, P.R. Newman ${ }^{3}$, T.C. Nicholls ${ }^{5}$, F. Niebergall ${ }^{11}$, C. Niebuhr ${ }^{10}$, O. Nix $^{14}$, G. Nowak ${ }^{6}$, T. Nunnemann ${ }^{12}$, J.E. Olsson ${ }^{10}$, D. Ozerov ${ }^{23}$, V. Panassik ${ }^{8}$, C. Pascaud $^{26}$, G.D. Patel ${ }^{18}$, E. Perez ${ }^{9}$, J.P. Phillips ${ }^{18}$, D. Pitzl ${ }^{10}$, R. Pöschl ${ }^{7}$, I. Potachnikova ${ }^{12}$, B. Povh ${ }^{12}$, K. Rabbertz ${ }^{1}$, G. Rädel ${ }^{1}$, J. Rauschenberger ${ }^{11}$, P. Reimer ${ }^{29}$, B. Reisert ${ }^{25}$, D. Reyna ${ }^{10}$, S. Riess ${ }^{11}$, C. Risler ${ }^{25}$, E. Rizvi ${ }^{3}$, P. Robmann ${ }^{37}$, R. Roosen ${ }^{4}$, A. Rostovtsev ${ }^{23}$, C. Royon $^{9}$, S. Rusakov ${ }^{24}$, K. Rybicki ${ }^{6}$, D.P.C. Sankey ${ }^{5}$, J. Scheins ${ }^{1}$, F.-P. Schilling ${ }^{13}$, P. Schleper ${ }^{10}$, D. Schmidt ${ }^{33}$, D. Schmidt ${ }^{10}$, S. Schmitt ${ }^{10}$, L. Schoeffel ${ }^{9}$, A. Schöning ${ }^{36}$, T. Schörner ${ }^{25}$, V. Schröder ${ }^{10}$, H.-C. Schultz-Coulon ${ }^{7}$, C. Schwanenberger ${ }^{10}$, K. Sedlák ${ }^{29}$, F. Sefkow ${ }^{37}$, V. Shekelyan ${ }^{25}$, I. Sheviakov ${ }^{24}$, L.N. Shtarkov ${ }^{24}$, P. Sievers ${ }^{13}$, Y. Sirois ${ }^{27}$, T. Sloan ${ }^{17}$, P. Smirnov ${ }^{24}$, V. Solochenko ${ }^{23, \dagger}$, Y. Soloviev ${ }^{24}$, V. Spaskov ${ }^{8}$, A. Specka ${ }^{27}$, H. Spitzer ${ }^{11}$, R. Stamen ${ }^{7}$, J. Steinhart ${ }^{11}$, B. Stella ${ }^{31}$, A. Stellberger ${ }^{14}$, J. Stiewe ${ }^{14}$, U. Straumann ${ }^{37}$, W. Struczinski ${ }^{2}$, M. Swart ${ }^{14}$, M. Taševský ${ }^{29}$, V. Tchernyshov ${ }^{23}$, S. Tchetchelnitski ${ }^{23}$, G. Thompson ${ }^{19}$, P.D. Thompson ${ }^{3}$, N. Tobien ${ }^{10}$, D. Traynor ${ }^{19}$, P. Truöl ${ }^{37}$, G. Tsipolitis ${ }^{10,38}$, I. Tsurin ${ }^{35}$, J. Turnau ${ }^{6}$, J.E. Turney ${ }^{19}$, E. Tzamariudaki ${ }^{25}$, S. Udluft $^{25}$, A. Usik $^{24}$, 
S. Valkár ${ }^{30}$, A. Valkárová ${ }^{30}$, C. Vallée ${ }^{22}$, P. Van Mechelen ${ }^{4}$, S. Vassiliev ${ }^{8}$, Y. Vazdik $^{24}$, A. Vichnevski ${ }^{8}$, K. Wacker ${ }^{7}$, R. Wallny ${ }^{37}$, T. Walter ${ }^{37}$, B. Waugh ${ }^{21}$, G. Weber ${ }^{11}$, M. Weber ${ }^{14}$, D. Wegener ${ }^{7}$, M. Werner ${ }^{13}$, G. White ${ }^{17}$, S. Wiesand ${ }^{33}$, T. Wilksen ${ }^{10}$, M. Winde ${ }^{35}$, G.-G. Winter ${ }^{10}$, Ch. Wissing ${ }^{7}$, M. Wobisch ${ }^{2}$, H. Wollatz ${ }^{10}$, E. Wünsch ${ }^{10}$, A.C. Wyatt ${ }^{21}$, J. Žáček ${ }^{30}$, J. Zálešák ${ }^{30}$, Z. Zhang ${ }^{26}$, A. Zhokin ${ }^{23}$, F. Zomer ${ }^{26}$, J. Zsembery ${ }^{9}$, and M. zur Nedden ${ }^{10}$

${ }^{1}$ I. Physikalisches Institut der RWTH, Aachen, Germany ${ }^{a}$

${ }^{2}$ III. Physikalisches Institut der RWTH, Aachen, Germany ${ }^{a}$

${ }^{3}$ School of Physics and Space Research, University of Birmingham, Birmingham, UK ${ }^{b}$

${ }^{4}$ Inter-University Institute for High Energies ULB-VUB, Brussels; Universitaire Instelling Antwerpen, Wilrijk; Belgium ${ }^{c}$

${ }^{5}$ Rutherford Appleton Laboratory, Chilton, Didcot, $U K^{b}$

${ }^{6}$ Institute for Nuclear Physics, Cracow, Poland ${ }^{d}$

${ }^{7}$ Institut für Physik, Universität Dortmund, Dortmund, Germany ${ }^{a}$

${ }^{8}$ Joint Institute for Nuclear Research, Dubna, Russia

${ }^{9}$ CEA, DSM/DAPNIA, CE-Saclay, Gif-sur-Yvette, France

${ }^{10}$ DESY, Hamburg, Germany ${ }^{a}$

${ }^{11}$ II. Institut für Experimentalphysik, Universität Hamburg, Hamburg, Germany ${ }^{a}$

${ }^{12}$ Max-Planck-Institut für Kernphysik, Heidelberg, Germany ${ }^{a}$

${ }^{13}$ Physikalisches Institut, Universität Heidelberg, Heidelberg, Germany ${ }^{a}$

${ }^{14}$ Kirchhoff-Institut für Physik, Universität Heidelberg, Heidelberg, Germany ${ }^{a}$

${ }^{15}$ Institut für experimentelle und angewandte Kernphysik, Universität Kiel, Kiel, Germany ${ }^{a}$

${ }^{16}$ Institute of Experimental Physics, Slovak Academy of Sciences, Košice, Slovak Republic ${ }^{e, f}$

${ }^{17}$ School of Physics and Chemistry, University of Lancaster, Lancaster, UK ${ }^{b}$

${ }^{18}$ Department of Physics, University of Liverpool, Liverpool, $U K^{b}$

${ }^{19}$ Queen Mary and Westfield College, London, $U K^{b}$

${ }^{20}$ Physics Department, University of Lund, Lund, Sweden ${ }^{g}$

${ }^{21}$ Physics Department, University of Manchester, Manchester, $U K^{b}$

${ }^{22}$ CPPM, CNRS/IN2P3 - Univ Mediterranee, Marseille - France

${ }^{23}$ Institute for Theoretical and Experimental Physics, Moscow, Russia

${ }^{24}$ Lebedev Physical Institute, Moscow, Russia ${ }^{e, h}$

${ }^{25}$ Max-Planck-Institut für Physik, München, Germany ${ }^{a}$

${ }^{26}$ LAL, Université de Paris-Sud, IN2P3-CNRS, Orsay, France

${ }^{27}$ LPNHE, Ecole Polytechnique, IN2P3-CNRS, Palaiseau, France

${ }^{28}$ LPNHE, Universités Paris VI and VII, IN2P3-CNRS, Paris, France

${ }^{29}$ Institute of Physics, Czech Academy of Sciences, Praha, Czech Republic ${ }^{e, i}$

${ }^{30}$ Faculty of Mathematics and Physics, Charles University, Praha, Czech Republice ${ }^{e, i}$

${ }^{31}$ Dipartimento di Fisica Università di Roma Tre and INFN Roma 3, Roma, Italy

${ }^{32}$ Paul Scherrer Institut, Villigen, Switzerland

${ }^{33}$ Fachbereich Physik, Bergische Universität Gesamthochschule Wuppertal, Wuppertal, Germany ${ }^{a}$

${ }^{34}$ Yerevan Physics Institute, Yerevan, Armenia

${ }^{35}$ DESY, Zeuthen, Germany ${ }^{a}$

${ }^{36}$ Institut für Teilchenphysik, ETH, Zürich, Switzerland ${ }^{j}$

${ }^{37}$ Physik-Institut der Universität Zürich, Zürich, Switzerland ${ }^{j}$ 
38 Also at Physics Department, National Technical University, Zografou Campus, GR-15773 Athens, Greece

${ }^{39}$ Also at Rechenzentrum, Bergische Universität Gesamthochschule Wuppertal, Germany

${ }^{40}$ Also at Institut für Experimentelle Kernphysik, Universität Karlsruhe, Karlsruhe, Germany

${ }^{41}$ Also at Dept. Fis. Ap. CINVESTAV, Mérida, Yucatán, México ${ }^{k}$

${ }^{42}$ Also at University of P.J. Šafárik, Košice, Slovak Republic

${ }^{43}$ Also at CERN, Geneva, Switzerland

${ }^{\dagger}$ Deceased

a Supported by the Bundesministerium für Bildung, Wissenschaft, Forschung und Technologie, FRG, under contract numbers 7AC17P, 7AC47P, 7DO55P, 7HH17I, 7HH27P, 7HD17P, 7HD27P, 7KI17I, 6MP17I and 7WT87P

${ }^{b}$ Supported by the UK Particle Physics and Astronomy Research Council, and formerly by the UK Science and Engineering Research Council

${ }^{c}$ Supported by FNRS-NFWO, IISN-IIKW

${ }^{d}$ Partially Supported by the Polish State Committee for Scientific Research, grant no. 2P0310318 and SPUB/DESY/P03/DZ-1/99, and by the German Federal Ministry of Education and Science, Research and Technology (BMBF)

${ }^{e}$ Supported by the Deutsche Forschungsgemeinschaft

${ }^{f}$ Supported by VEGA SR grant no. 2/5167/98

${ }^{g}$ Supported by the Swedish Natural Science Research Council

${ }^{h}$ Supported by Russian Foundation for Basic Researc grant no. 96-02-00019

${ }^{i}$ Supported by GA AV ČR grant no. A1010821

${ }^{j}$ Supported by the Swiss National Science Foundation

${ }^{k}$ Supported by CONACyT 


\section{Introduction}

Inclusive Deep Inelastic Scattering (DIS) has long been used as a sensitive probe of proton structure and Quantum Chromodynamics (QCD). Since 1992 the experiments H1 and ZEUS have used the colliding lepton-proton beams of the HERA accelerator to further extend the phase space of such measurements into new kinematic regions of large four-momentum transfer squared $Q^{2}$ and small $x$, where $x$ is the Bjorken scaling variable. The large integrated luminosity collected by the experiments has allowed measurements to be made in the very high $Q^{2}$ range up to $30000 \mathrm{GeV}^{2}$. In the region where $Q^{2} \simeq M_{Z}^{2}$ or $M_{W}^{2}$, the $Z^{0}$ and $W^{ \pm}$boson masses squared, the effects of the electroweak sector of the Standard Model can be tested in DIS. In addition any deviation from the prediction observed at the highest $Q^{2}$, where the smallest distance scales of proton structure are probed may indicate new physics, beyond the Standard Model.

Both contributions to DIS, neutral current (NC) interactions $e p \rightarrow e X$ and charged current (CC) interactions $e p \rightarrow \nu X$, can be measured at HERA and give complementary information on the QCD and electroweak parts of the Standard Model. The cross sections are defined in terms of the three kinematic variables $Q^{2}, x$, and $y$, where $y$ quantifies the inelasticity of the interaction. The kinematic variables are related via $Q^{2}=s x y$, where $\sqrt{s}$ is the $e p$ centre-of-mass energy.

In this paper we report on $\mathrm{NC}$ and $\mathrm{CC}$ cross section measurements using $e^{-} p$ data taken during 1998 and 1999 with a proton beam energy of $E_{p}=920 \mathrm{GeV}$ and an electron beam energy of $E_{e}=27.6 \mathrm{GeV}$, leading to a centre-of-mass energy of $\sqrt{s} \approx 320 \mathrm{GeV}$. The integrated luminosity of this sample is $16.4 \mathrm{pb}^{-1}$ which represents an increase in integrated luminosity by a factor of approximately 20 compared with previous HERA measurements of $e^{-} p$ cross sections [1].

The results are compared with $\mathrm{NC}$ and $\mathrm{CC}$ measurements of $e^{+} p$ scattering from $\mathrm{H} 1$ [2] and ZEUS [3] taken at a lower centre-of-mass energy of $\sqrt{s} \approx 300 \mathrm{GeV}$. The $e^{+} p$ and $e^{-} p \mathrm{NC}$ data from $\mathrm{H} 1$ are used to make the first measurements of the generalised structure function $x \tilde{F}_{3}$ in the very high $Q^{2}$ domain $\left(Q^{2}>1000 \mathrm{GeV}^{2}\right)$.

\section{Neutral and Charged Current Cross Sections}

The DIS cross sections $\sigma_{N C(C C)}$ for NC and CC processes in $e^{ \pm} p$ collisions may be factorised as $\sigma_{N C(C C)}=\sigma_{N C(C C)}^{B o r n}\left(1+\delta_{N C(C C)}^{q e d}\right)\left(1+\delta_{N C(C C)}^{w e a k}\right)$ where $\sigma_{N C(C C)}^{B o r n}$ is the Born cross section and $\delta_{N C(C C)}^{q e d}$ and $\delta_{N C(C C)}^{w e a k}$ are the QED and weak radiative corrections respectively.

The NC cross section for the process $e^{ \pm} p \rightarrow e^{ \pm} X$ with unpolarised beams and corrected for $\mathrm{QED}$ radiative effects is given by

$$
\frac{\mathrm{d}^{2} \sigma_{N C}^{ \pm}}{\mathrm{d} x \mathrm{~d} Q^{2}}=\frac{2 \pi \alpha^{2}}{x Q^{4}}\left[Y_{+} \tilde{F}_{2} \mp Y_{-} x \tilde{F}_{3}-y^{2} \tilde{F}_{L}\right]\left(1+\delta_{N C}^{w e a k}\right)
$$

where $\alpha$ is the fine structure constant taken to be $\alpha \equiv \alpha\left(Q^{2}=0\right)$. The $\delta_{N C}^{\text {weak }}$ corrections are defined in [円] with the Fermi coupling constant, $G_{F}$, and $M_{Z}$ as the other main electroweak inputs. 
The helicity dependences of the electroweak interactions are contained in $Y_{ \pm} \equiv 1 \pm(1-y)^{2}$. The generalised structure functions $\tilde{F}_{2}$ and $x \tilde{F}_{3}$ can be decomposed as follows [5]

$$
\begin{array}{ccc}
\tilde{F}_{2} & \equiv F_{2}-v_{e} \frac{\kappa_{w} Q^{2}}{\left(Q^{2}+M_{Z}^{2}\right)} F_{2}^{\gamma Z}+\left(v_{e}^{2}+a_{e}^{2}\right)\left(\frac{\kappa_{w} Q^{2}}{Q^{2}+M_{Z}^{2}}\right)^{2} F_{2}^{Z} \\
x \tilde{F}_{3} & \equiv \quad-a_{e} \frac{\kappa_{w} Q^{2}}{\left(Q^{2}+M_{Z}^{2}\right)} x F_{3}^{\gamma Z}+\left(2 v_{e} a_{e}\right)\left(\frac{\kappa_{w} Q^{2}}{Q^{2}+M_{Z}^{2}}\right)^{2} x F_{3}^{Z},
\end{array}
$$

where $\kappa_{w}^{-1}=4 \frac{M_{W}^{2}}{M_{Z}^{2}}\left(1-\frac{M_{W}^{2}}{M_{Z}^{2}}\right)$ in the on-shell scheme [6] and $M_{W}$ is defined in terms of the electroweak inputs. The quantities $v_{e}$ and $a_{e}$ are the vector and axial couplings of the electron to the $Z^{0}$ [6]. The electromagnetic structure function $F_{2}$ originates from photon exchange only. The functions $F_{2}^{Z}$ and $x F_{3}^{Z}$ are the contributions to $\tilde{F}_{2}$ and $x \tilde{F}_{3}$ from $Z^{0}$ exchange and the functions $F_{2}^{\gamma Z}$ and $F_{3}^{\gamma Z}$ are the contribution from $\gamma Z^{0}$ interference. The purely longitudinal structure function $\tilde{F}_{L}$ may be decomposed in a manner similar to $\tilde{F}_{2}$. Its contribution is significant only at high $y$ and is expected to diminish with increasing $Q^{2}$.

Over most of the kinematic domain at HERA the dominant contribution to the cross section comes from the electromagnetic structure function $F_{2}$. Only at large values of $Q^{2}$ do the contributions due to $Z^{0}$ exchange become important. For longitudinally unpolarised lepton beams $\tilde{F}_{2}$ is the same for electron and for positron scattering, while the $x \tilde{F}_{3}$ contribution changes sign as can be seen in eq. 1. In $e^{-} p$ scattering, due to the positive interference between photon and $Z^{0}$ exchange, the Standard Model cross section is larger than that calculated in a model which includes only photon exchange. Conversely for $e^{+} p$ scattering, within the HERA kinematic domain, the negative interference arising from the $x \tilde{F}_{3}$ term, results in a cross section that is smaller than in the photon exchange only model.

In the quark parton model (QPM) the structure functions $F_{2}, F_{2}^{\gamma Z}$ and $F_{2}^{Z}$ are related to the sum of the quark and anti-quark densities

$$
\left[F_{2}, F_{2}^{\gamma Z}, F_{2}^{Z}\right]=x \sum_{q}\left[e_{q}^{2}, 2 e_{q} v_{q}, v_{q}^{2}+a_{q}^{2}\right]\{q+\bar{q}\}
$$

and the structure functions $x F_{3}^{\gamma Z}$ and $x F_{3}^{Z}$ to the difference between quark and anti-quark densities

$$
\left[x F_{3}^{\gamma Z}, x F_{3}^{Z}\right]=x \sum_{q}\left[2 e_{q} a_{q}, 2 v_{q} a_{q}\right]\{q-\bar{q}\} .
$$

The functions $q$ and $\bar{q}$ are the parton distribution functions (PDFs) for quarks and anti-quarks, $e_{q}$ is the charge of quark $q$ in units of the electron charge and $v_{q}$ and $a_{q}$ are the vector and axial-vector couplings of the quarks.

For CC interactions the cross section corrected for QED radiative effects may be expressed as

$$
\begin{aligned}
\frac{\mathrm{d}^{2} \sigma_{\mathrm{CC}}^{ \pm}}{\mathrm{d} x \mathrm{~d} Q^{2}} & =\frac{G_{F}^{2} M_{W}^{4}}{2 \pi x} \frac{1}{\left(Q^{2}+M_{W}^{2}\right)^{2}} \phi_{\mathrm{CC}}^{ \pm}\left(1+\delta_{C C}^{\text {weak }}\right) \\
\text { with } \quad \phi_{\mathrm{CC}}^{ \pm} & =\frac{1}{2}\left(Y_{+} \tilde{W}_{2}^{ \pm} \mp Y_{-} x \tilde{W}_{3}^{ \pm}-y^{2} \tilde{W}_{L}^{ \pm}\right),
\end{aligned}
$$


where $\delta_{C C}^{w e a k}$ are the $\mathrm{CC}$ weak radiative corrections. The structure functions for CC interactions $\tilde{W}_{L}, \tilde{W}_{2}$, and $x \tilde{W}_{3}$ are defined in analogy to the NC case. In the QPM, neglecting contributions from the $t$ and $b$ quarks, the structure function term for $e^{ \pm} p \rightarrow \nu X$ can be written as

$$
\phi_{\mathrm{CC}}^{+}=x\left[(\bar{u}+\bar{c})+(1-y)^{2}(d+s)\right], \quad \phi_{\mathrm{CC}}^{-}=x\left[(u+c)+(1-y)^{2}(\bar{d}+\bar{s})\right]
$$

where $u, c, d, s$ are the quark distributions and $\bar{u}, \bar{c}, \bar{d}, \bar{s}$ are the anti-quark distributions.

The measured cross sections presented in section 4 , in which the effects of QED radiation have been corrected for, correspond to the differential cross sections $\mathrm{d}^{2} \sigma_{N C(C C)} / \mathrm{d} x \mathrm{~d} Q^{2}$ defined in eq. 1 and 6. The corrections $\left(\delta_{N C(C C)}^{q e d}\right)$ are defined in [2] and were calculated using the program HERACLES [7] as implemented in DJANGO [8] and verified with the analytic program HECTOR [9]. The radiative corrections due to the exchange of two or more photons between the lepton and the quark lines, which are not included in DJANGO, vary with the polarity of the lepton beam. This variation is small compared to the quoted errors and is neglected. The weak corrections $\left(\delta_{N C(C C)}^{w e a k}\right)$, are typically of the order of $1 \%$ and have not been applied to the measured cross sections, but are applied to determine the electromagnetic structure function $F_{2}$ and the $\mathrm{CC}$ structure function term $\phi_{C C}$.

It is convenient to derive the $\mathrm{NC}$ and $\mathrm{CC}$ "reduced cross sections" in which the dominant part of the $Q^{2}$ dependence of $\mathrm{d}^{2} \sigma / \mathrm{d} x \mathrm{~d} Q^{2}$ due to the boson propagators is removed. The reduced cross sections for $\mathrm{NC}$ and $\mathrm{CC}$ are defined as

$$
\tilde{\sigma}_{N C}\left(x, Q^{2}\right) \equiv \frac{1}{Y_{+}} \frac{Q^{4} x}{2 \pi \alpha^{2}} \frac{\mathrm{d}^{2} \sigma_{N C}}{\mathrm{~d} x \mathrm{~d} Q^{2}}, \quad \tilde{\sigma}_{C C}\left(x, Q^{2}\right) \equiv \frac{2 \pi x}{G_{F}^{2}}\left(\frac{M_{W}^{2}+Q^{2}}{M_{W}^{2}}\right)^{2} \frac{\mathrm{d}^{2} \sigma_{C C}}{\mathrm{~d} x \mathrm{~d} Q^{2}} .
$$

The expression used to extract the electromagnetic structure function $F_{2}$ from the measured NC reduced cross section is:

$$
\tilde{\sigma}_{N C}=F_{2}\left(1+\Delta_{F_{2}}+\Delta_{F_{3}}+\Delta_{F_{L}}\right)\left(1+\delta_{N C}^{\text {weak }}\right)=F_{2}\left(1+\Delta_{\text {all }}\right),
$$

where the correction terms $\Delta_{F_{2}}$ and $\Delta_{F_{3}}$ account for the relative contribution of pure $Z^{0}$ exchange and photon- $Z^{0}$ interference to $\tilde{F}_{2}$ and $x \tilde{F}_{3}$, and $\Delta_{F_{L}}$ originates from the longitudinal structure function $\tilde{F}_{L}$.

\section{Experimental Technique}

\subsection{H1 Apparatus}

The co-ordinate system of $\mathrm{H} 1$ is defined such that the positive $z$ axis is in the direction of the incident proton beam. The polar angle $\theta$ is then defined with respect to the positive $z$ axis which defines the forward direction. The detector components most relevant to this analysis are the LAr calorimeter, which measures the angles and energies of particles over the range $4^{\circ}<\theta<154^{\circ}$, a lead-fibre calorimeter (SPACAL) covering the range $153^{\circ}<\theta<177^{\circ}$ and the inner tracking detectors which measure the angles and momenta of charged particles over the range $7^{\circ}<\theta<165^{\circ}$. In addition the PLUG calorimeter covers the range $0.7^{\circ}<\theta<3.3^{\circ}$. A full description of the $\mathrm{H} 1$ detector can be found in [10] and [11].

\footnotetext{
${ }^{1}$ The explicit definitions are given in eq. 17 of [2].
} 


\subsection{Monte Carlo Generation Programs}

In order to determine acceptance corrections and background contributions for the DIS cross section measurements, the detector response to events produced by various Monte Carlo (MC) generation programs is simulated in detail using a program based on GEANT [12]. These simulated events are then subjected to the same reconstruction and analysis chain as the real data.

DIS processes are generated using the DJANGO [8] program which is based on HERACLES [7] for the electroweak interaction and on LEPTO [13], using the colour dipole model as implemented in ARIADNE [14] to generate the QCD dynamics. The JETSET program is used for the hadron fragmentation [15]. The simulated events are produced with PDFs from the next to leading order QCD fit [2] performed on fixed target data from NMC [16] and BCDMS [17], and $\mathrm{H} 1 e^{+} p$ data [2]. The fit gives a good description of the data and is referred to as the "H1 97 PDF Fit" in the following.

The dominant $e p$ background contribution to NC and CC processes is due to photoproduction $(\gamma p)$ events. These are simulated using the PYTHIA [18] generator with GRV leading order parton distribution functions for the proton and photon [19].

\subsection{Kinematic Reconstruction and Calibration}

The NC event kinematics are reconstructed using the $e \Sigma$ method [20], which uses the energy $E_{e}^{\prime}$ and polar angle $\theta_{e}$ of the scattered electron and the quantity $\Sigma=\sum_{i}\left(E_{i}-p_{z, i}\right)$, where the summation is performed over all objects in the hadronic final state assuming particles of zero rest mass. This method gives good resolution in $x$ and $Q^{2}$ throughout the kinematic range.

The CC event kinematics can only be determined with the hadron method ( $h$ method) [21], which uses $\Sigma$ and the hadronic transverse momentum $P_{T, h}=\sqrt{\left(\sum_{i} p_{x, i}\right)^{2}+\left(\sum_{i} p_{y, i}\right)^{2}}$, where the summation is performed over all hadronic final state particles.

The accessible kinematic range depends on the resolution of the reconstructed kinematics and is determined by requiring the purity and stability of any $\left(x, Q^{2}\right)$ bin to be larger than $30 \%$. The stability (purity) is defined as the fraction of events which originate from a bin and which are reconstructed in it, divided by the number of generated (reconstructed) events in that bin.

The electromagnetic and hadronic response of the detector is calibrated using the analysis described in [2]. The procedure is found to give an excellent description of the detector response by the simulation. The hadronic final state is measured by combining calorimeter energy deposits (clusters) with low momentum tracks. Isolated, low energy calorimeter clusters are classified as noise and are not included in the determination of the hadronic final state.

\subsection{Selection of NC Events}

High $Q^{2} \mathrm{NC}$ events are selected by requiring that the event has a compact electromagnetic cluster in the LAr calorimeter, taken to be the scattered electron, in addition to an interaction 
H1 Neutral Current Data
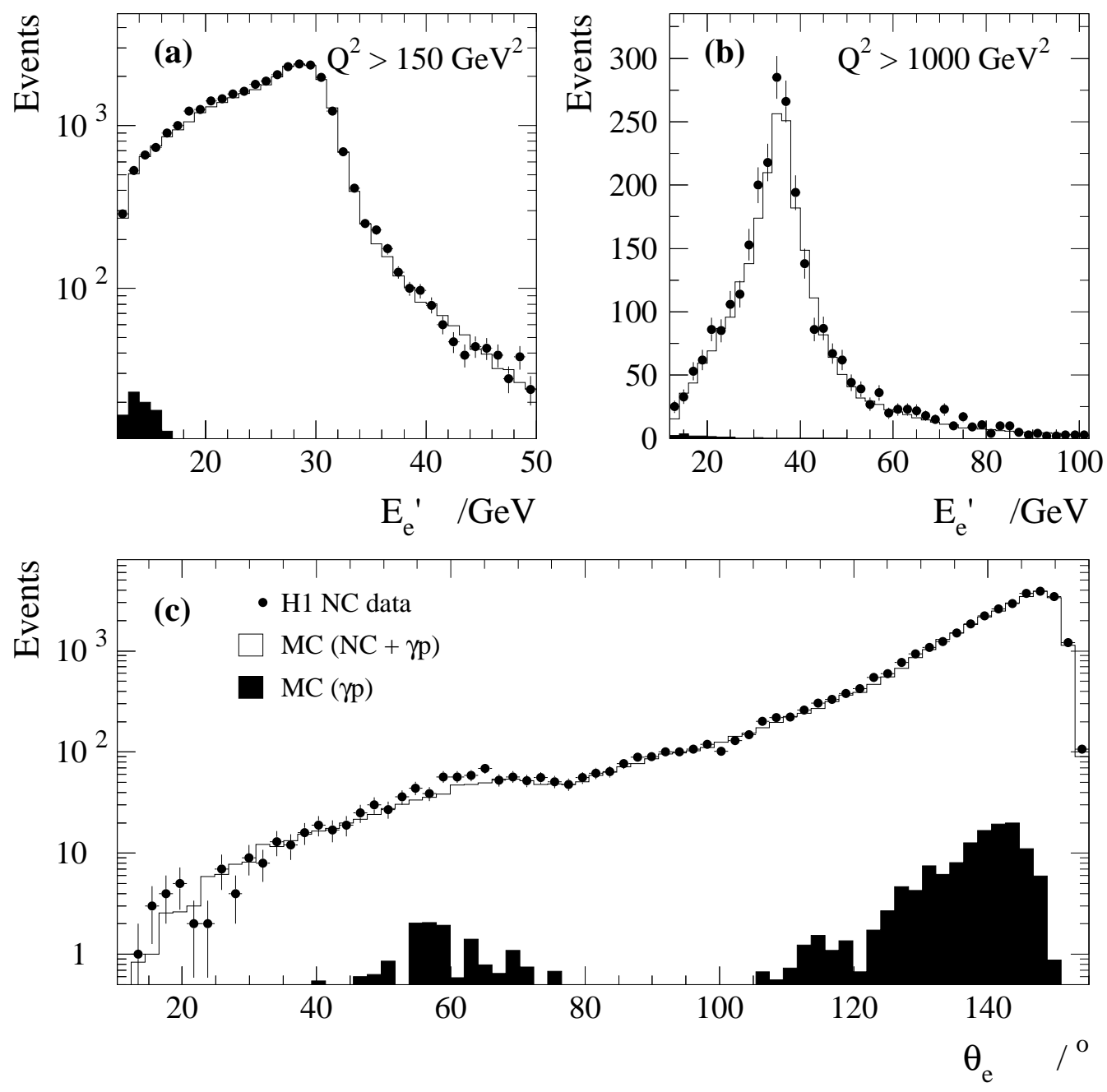

Figure 1: Distributions of (a) $E_{e}^{\prime}$ for $Q^{2}>150 \mathrm{GeV}^{2}$, (b) $E_{e}^{\prime}$ for $Q^{2}>1000 \mathrm{GeV}^{2}$ and (c) $\theta_{e}$ for NC data (solid points) and simulation (solid line). The filled histograms show the photoproduction contribution.

vertex position within $\pm 35 \mathrm{~cm}$ of its nominal position along the $z$ axis. The scattered electron energy and polar angle are determined from the calorimeter cluster. For $Q^{2}$ less than $890 \mathrm{GeV}^{2}$ corresponding to a bin boundary, the requirement $y_{e}<0.63$, where $y$ is determined from the electron method $\left(y_{e}=1-\frac{E_{e}^{\prime}}{E_{e}} \sin ^{2} \frac{\theta_{e}}{2}\right.$ ), ensures that the measurement is confined to the region where the trigger efficiency is greater than $99.5 \%$. Fiducial cuts are made to remove local regions where the electromagnetic shower of the scattered electron is not fully contained in the calorimeter, and where the trigger is not fully efficient. Further details are given in [22].

The most significant background in the NC sample arises from photoproduction interactions where hadronic final state particles are misidentified as the scattered electron. This background is suppressed by requiring that $\Sigma+E_{e}^{\prime}\left(1-\cos \theta_{e}\right)>35 \mathrm{GeV}, y_{e}<0.9$ and that there is an extrapolated track with a distance of closest approach to the cluster of less than $12 \mathrm{~cm}$. This latter requirement is only applied for $\theta_{e} \geq 40^{\circ}$, where the track reconstruction efficiency is 
greater than $97 \%$.

The final sample of selected NC data consists of about 40000 events. The scattered electron energy spectrum of the data is compared to simulation in fig. 11(a) for $Q^{2}>150 \mathrm{GeV}^{2}$. The simulation is in good agreement with the data, particularly for the region $E_{e}^{\prime}>26 \mathrm{GeV}$ which is sensitive to the details of the calibration and resolution simulation. In fig. 1(b) the $E_{e}^{\prime}$ spectrum is also shown for $Q^{2}>1000 \mathrm{GeV}^{2}$. The distribution of the electron polar angle, $\theta_{e}$, is presented in fig. 11(c) where the data are well described by the simulation across the full range of $\theta_{e}$. The filled histogram shows the photoproduction contribution which falls rapidly with decreasing $\theta_{e}$, in part due to the requirement $y<0.63$. At higher $Q^{2}$, and correspondingly lower $\theta_{e}$, the accessible $y$ range is extended to $y<0.9$ and is responsible for the extra background contribution visible around $\theta_{e} \sim 60^{\circ}$.

\subsection{Selection of CC Events}

The selection of CC events is based on the expectation that the unseen neutrino will result in an observed imbalance in the transverse momentum, $P_{T, h}$. A requirement that $P_{T, h} \geq 12 \mathrm{GeV}$ is therefore made. In addition the event must have a reconstructed vertex within $\pm 35 \mathrm{~cm}$ of its nominal position.

The non-ep background in the $\mathrm{CC}$ sample is rejected using timing requirements and a set of topological finders based on track and calorimeter patterns consistent with cosmic events or particles from the halo of the proton beam [23]. The remaining ep background, which is dominantly due to photoproduction events, is suppressed using the ratio $V_{a p} / V_{p}$ and the difference in azimuth between $\vec{P}_{T, h}$ measured in the main detector and the PLUG calorimeter, $\Delta \phi_{h, \text { PLUG. }}$. The quantities $V_{p}$ and $V_{a p}$ are respectively the transverse energy flow parallel and anti-parallel to $\vec{P}_{T, h}$; they are determined from the transverse momentum vectors $\vec{P}_{T, i}$ of all the particles $i$ which belong to the hadronic final state according to

$$
\begin{gathered}
V_{p}=\sum_{i} \frac{\vec{P}_{T, h} \cdot \vec{P}_{T, i}}{P_{T, h}} \text { for } \vec{P}_{T, h} \cdot \vec{P}_{T, i}>0 \\
V_{a p}=-\sum_{i} \frac{\vec{P}_{T, h} \cdot \vec{P}_{T, i}}{P_{T, h}} \text { for } \vec{P}_{T, h} \cdot \vec{P}_{T, i}<0 .
\end{gathered}
$$

$\mathrm{CC}$ events tend to have little energy in the hemisphere around the direction of the neutrino and consequently have small values of $V_{a p} / V_{p}$. Conversely the energy is more isotropic in photoproduction events which generally have higher values of $V_{a p} / V_{p}$. One of the main types of photoproduction background arises from events that contain a jet at low polar angle such that not all of the energy is recorded in the main detector, resulting in a measured imbalance in transverse momentum. This missing momentum is, however, generally tagged in the PLUG calorimeter, with such events having values of $\Delta \phi_{h, \mathrm{PLUG}}$ close to $180^{\circ}$. The two anti-photoproduction criteria are combined with $P_{T, h}$ so as to maximise the background rejection whilst still retaining a high efficiency for $\mathrm{CC}$ events.

For $P_{T, h}<25 \mathrm{GeV}$ a $P_{T, h}$ dependent cut is applied in the $\Delta \phi_{h, \text { PLUG }}-V_{a p} / V_{p}$ plane, whereas for $P_{T, h}>25 \mathrm{GeV}$, the cut is simplified to $V_{a p} / V_{p}<0.2$. The cut gains a factor of two 
improvement in background rejection whilst retaining a similar selection efficiency compared to the cut $V_{a p} / V_{p}<0.15$ used in previous analyses [2]. Further details are available in [24].

In order to restrict the measurement to a region where the kinematic reconstruction is optimal the events are required to have $y_{h}=\Sigma / 2 E_{e}<0.85$. The CC trigger efficiency is determined using NC events in which all information associated to the scattered electron is removed. This method gives a precise measure of the efficiency which is found to be $63 \%$ at $Q^{2}=300 \mathrm{GeV}^{2}$ and reaches $98 \%$ at $Q^{2}=5000 \mathrm{GeV}^{2}$. The measurement is restricted to the region where the trigger efficiency is everywhere greater than $40 \%$ by demanding $y_{h}>0.03$.

The final CC data sample contains about 700 events. The data and simulation are compared in fig. 2 for the $P_{T, h}$ and $y_{h}$ spectra. In both cases the simulation gives a good description of the data.

H1 Charged Current Data
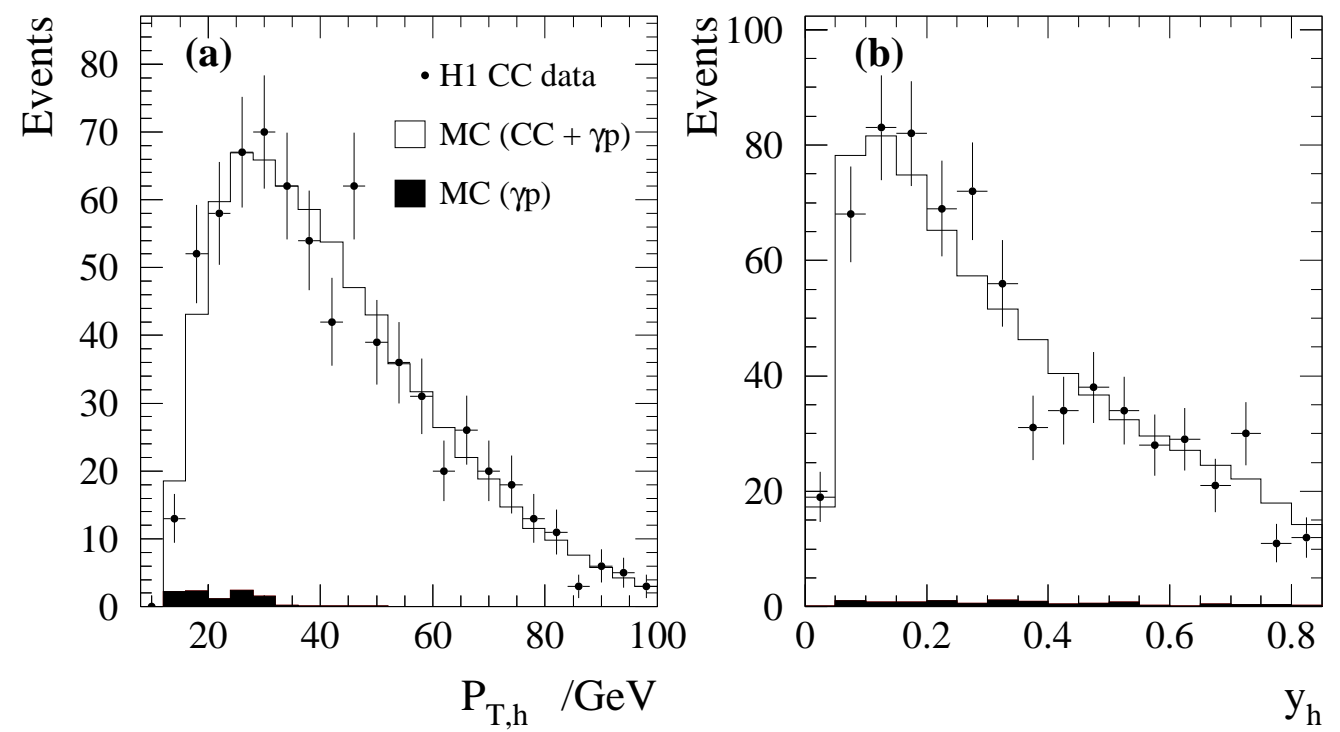

Figure 2: Distributions of (a) $P_{T, h}$ and (b) $y_{h}$ for CC data (solid points) and simulation (solid line). The filled histograms show the photoproduction contribution.

\subsection{Cross Section Determination}

The photoproduction background is estimated using simulated events from the PYTHIA generator and checked with a subsample of events which contain an electron tagged at small scattering angles. The contribution to the NC cross sections is never more than 5\% at the highest $y$ and negligible elsewhere. The background in the CC sample is at most $13 \%$ at the highest $y$ at low $Q^{2}$ and below $1 \%$ for $Q^{2}>1000 \mathrm{GeV}^{2}$. The background is statistically subtracted for each measurement bin.

The measured distributions are corrected for detector acceptance, migrations, the effects of QED radiation using the DJANGO simulation, and converted to cross sections at a specified bin centre by comparison with the H1 97 PDF Fit [2]. 


\subsection{Systematic Errors}

The uncertainties on the measurement lead to systematic errors on the cross sections, which can be split into bin to bin correlated or uncorrelated parts. All the correlated systematic errors are found to be symmetric to a good approximation and are assumed so in the following. The total systematic error is formed by adding the individual errors in quadrature. The correlated and the uncorrelated systematic errors of the $\mathrm{NC}$ and $\mathrm{CC}$ cross section measurements are given in tables 8 and 9 and their origin is discussed below.

- An uncertainty on the electron energy of $1.5 \%$ if the $z$ impact position of the electron at the calorimeter surface $\left(z_{i m p}\right)$ is in the backward part $\left(z_{i m p}<-150 \mathrm{~cm}\right)$, of $1 \%$ in the region $-150<z_{i m p}<20 \mathrm{~cm}$, of $2 \%$ for $20<z_{i m p}<110 \mathrm{~cm}$ and of $3 \%$ in the forward part $\left(z_{i m p}>110 \mathrm{~cm}\right)$. These uncertainties are obtained by the quadratic sum of an uncorrelated uncertainty and a bin to bin correlated uncertainty. The correlated uncertainty comes mainly from the potential bias of the calibration method and is estimated to be $0.5 \%$ in the whole LAr calorimeter. It results in a correlated systematic error on the NC cross section which is $\approx 3 \%$ at low $y$ and $Q^{2} \lesssim 1000 \mathrm{GeV}^{2}$.

- A correlated uncertainty of $3 \mathrm{mrad}$ on the determination of the electron polar angle. This leads to an uncertainty on the NC reduced cross section which does not exceed $5 \%$.

- A $2 \%$ uncertainty on the hadronic energy in the LAr calorimeter which is obtained from the quadratic sum of an uncorrelated systematic uncertainty of $1.7 \%$ and a correlated uncertainty of $1 \%$ originating from the calibration method and from the uncertainty of the reference scale $\left(P_{T, e}\right)$. The resulting correlated systematic error increases at low $y$, and is typically $\lesssim 4 \%$.

- A correlated $25 \%$ uncertainty on the amount of noise energy subtracted in the LAr calorimeter, which gives rise to a correlated systematic error at low $y$, e.g. $\simeq 5 \%$ at $x=0.65$ and $Q^{2} \leq 2000 \mathrm{GeV}^{2}$ in the NC measurements.

- A $7 \%(3 \%)$ uncertainty on the energy of the hadronic final state measured in the SPACAL (tracking system). The influence on the cross section is small compared to the uncorrelated uncertainty of the LAr calorimeter energy, and so the three contributions (LAr, SPACAL, tracks) have been added quadratically, giving rise to the uncorrelated hadronic error which is given in table 8 for the $\mathrm{NC}$ data and in table 9 for the $\mathrm{CC}$ data.

- The correlated error due to the uncertainty of the efficiency of the anti-photoproduction cut is estimated by varying the quantity $V_{a p} / V_{p}$ by \pm 0.02 . This leads to a maximum error at low $P_{T, h}$ in the $\mathrm{CC}$ analysis of $12 \%$.

- The $30 \%$ uncertainty on the subtracted photoproduction background results in a correlated systematic error always smaller than $5 \%$ in any bin, both for the NC and CC measurements.

The following uncertainties, which lead to equivalent uncorrelated systematic errors on the cross sections, have also been taken into account as listed below. 
- A $2 \%$ error originating from the electron identification efficiency in the $\mathrm{NC}$ analysis.

- A $1 \%$ error on the efficiency of the track-cluster link requirement in the $\mathrm{NC}$ analysis.

- A $0.5 \%$ error on the trigger efficiency in the $\mathrm{NC}$ analysis, and from 2 to $6 \%$ in the $\mathrm{CC}$ analysis.

- An error of $1 \%(\mathrm{NC}), 3 \%(\mathrm{CC})$ on the cross section originating from the QED radiative corrections.

- A $3 \%$ error on the efficiency of the non-ep background finders in the CC analysis.

- A $2 \%$ error $(5 \%$ for $y<0.1)$ on the vertex finding efficiency for CC events.

Further details can be found in [22, 23]. Overall the typical total systematic error for the NC (CC) double differential cross section is about $5 \%(12 \%)$. For the $\mathrm{d} \sigma_{N C(C C)} / \mathrm{d} Q^{2}$ measurements, the equivalent error is typically $3 \%(8 \%)$. In addition a $1.8 \%$ normalisation error, due to the luminosity uncertainty, must be considered, but is not included in the systematic error of the measurements given in the tables, or shown in the figures.

\section{Results}

\subsection{NC and CC Cross Sections $\mathrm{d} \sigma / \mathrm{dx}$}

The dependence of the $\mathrm{NC}$ cross sections as a function of $x$ is shown in fig. 3 for both $e^{-} p$ and $e^{+} p$ scattering. The data are shown for $y<0.9$ and $Q^{2}>1000 \mathrm{GeV}^{2}$ in fig. $3(\mathrm{a}, \mathrm{b})$ and listed in table 11. The data for $y<0.9$ and $Q^{2}>10000 \mathrm{GeV}^{2}$ are shown in fig. $3(\mathrm{c}, \mathrm{d})$ and listed in table 2 . Fig. 3 also shows the expectation from the Standard Model, derived from the H1 97 PDF Fit to the $\mathrm{H} 1 e^{+} p$ data [2]. The data are also compared to a model of pure photon exchange, where the effects of $Z^{0}$ exchange are neglected.

The cross sections for $Q^{2}>1000 \mathrm{GeV}^{2}$ are in agreement with the Standard Model, although for $e^{-} p$ they have a tendency to be larger at low $x$ and are also found to be larger compared to the pure photon exchange model. For $Q^{2}>10000 \mathrm{GeV}^{2}$ the $e^{-} p$ cross section is found to be approximately a factor of four larger than for $e^{+} p$ scattering at $x \simeq 0.2$ of which only $12 \%$ is due to the different centre-of-mass energies. The $e^{-} p$ cross sections are seen to lie above the pure photon exchange model, whereas $e^{+} p$ cross sections lie below it. Both measurements agree with the Standard Model.

The observed difference at high $Q^{2}$ between the $e^{+} p$ and $e^{-} p$ data and between both sets of data and the pure photon exchange model is understood in the Standard Model by the effects of $Z^{0}$ exchange. It can be deduced from eq. 2 that the generalised structure function $\tilde{F}_{2}$ is always larger than the electromagnetic structure function $F_{2}$. Furthermore since $|v| \ll a^{2} \kappa_{w}$ the increase at large $Q^{2}$ is expected to be mainly due to the $Z^{0}$ exchange contribution $F_{2}^{Z}$. Conversely the main contribution to $x \tilde{F}_{3}$ is from the photon- $Z^{0}$ interference term. For $e^{-} p$ scattering the cross section is enhanced relative to pure photon exchange, since the contributions from $F_{2}^{Z}$ 

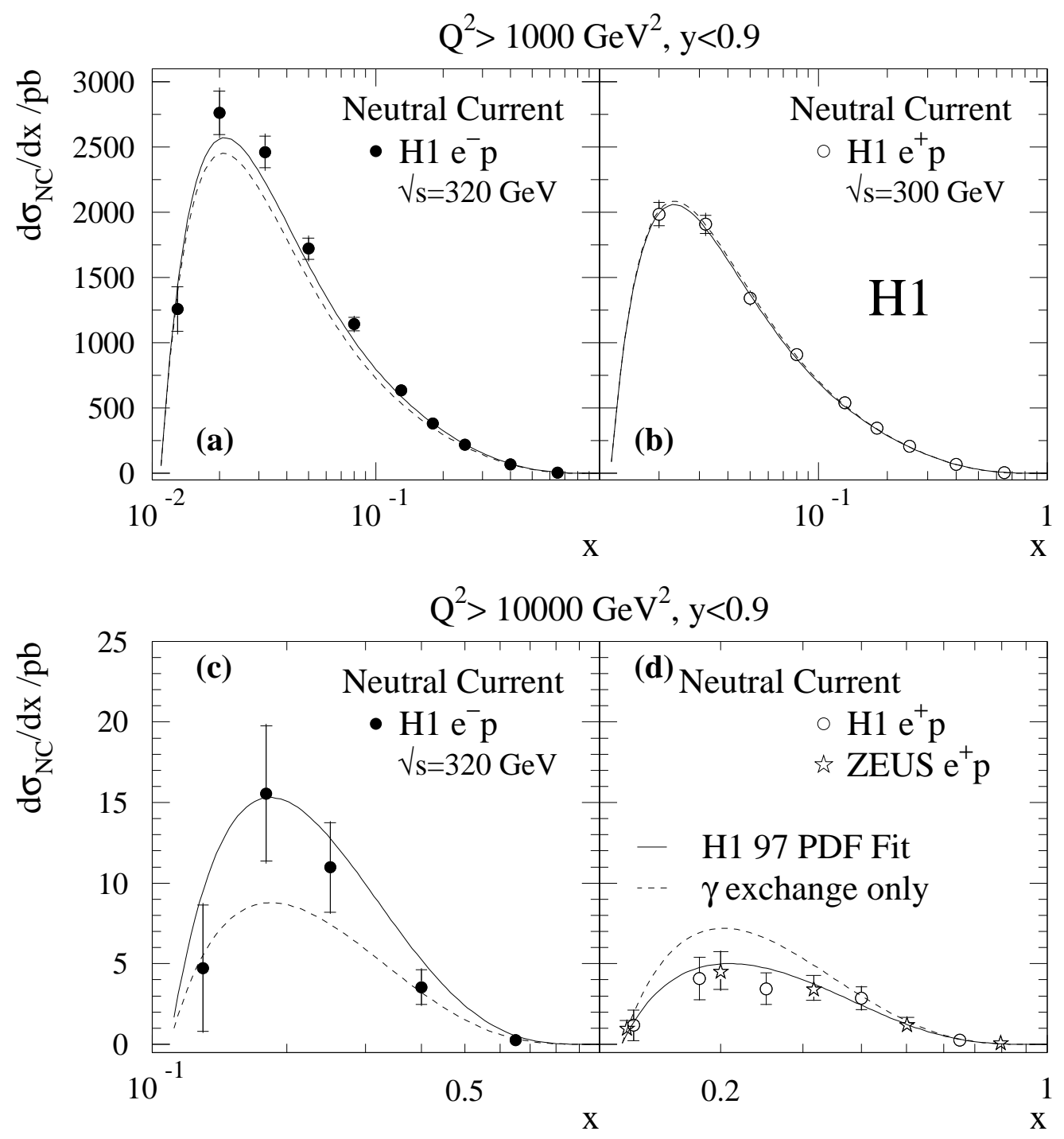

Figure 3: The NC cross sections $\mathrm{d} \sigma_{\mathrm{NC}} / \mathrm{d} x$ for the $e^{-} p$ data are shown in (a) for $Q^{2}>$ $1000 \mathrm{GeV}^{2}$ and in (c) for $Q^{2}>10000 \mathrm{GeV}^{2}$. The $\mathrm{H} 1 e^{+} p$ cross sections [2] are shown in (b) and (d) for $Q^{2}>1000 \mathrm{GeV}^{2}$ and $10000 \mathrm{GeV}^{2}$ respectively. In addition the ZEUS $e^{+} p$ data [3] are shown for $Q^{2}>10000 \mathrm{GeV}^{2}$. The solid curves show the Standard Model expectation based on the H1 97 PDF Fit. The dashed curves show the contribution of photon exchange only. All cross sections are shown for $y<0.9$. The inner error bars represent the statistical error, and the outer error bars show the total error. The normalisation uncertainties are not included in the error bars.

and $x \tilde{F}_{3}$ are both positive (see eq. 1). For $e^{+} p$ scattering $F_{2}^{Z}$ gives a positive contribution and $x \tilde{F}_{3}$ gives a negative contribution. In the HERA kinematic range the contribution from $x \tilde{F}_{3}$ is larger than that from $F_{2}^{Z}$ and so the resulting cross section is smaller than that from pure photon exchange.

The CC cross section $\mathrm{d} \sigma / \mathrm{d} x$, is measured for $Q^{2}>1000 \mathrm{GeV}^{2}$ and $y<0.9$ and is shown in fig. 7 and listed in table 3 . A correction has been made for the cuts $0.03<y<0.85$ using the H1 97 PDF Fit and is given in table 3. Fig. 7 also shows the corresponding measurement in $e^{+} p$ 
scattering and the Standard Model expectation.

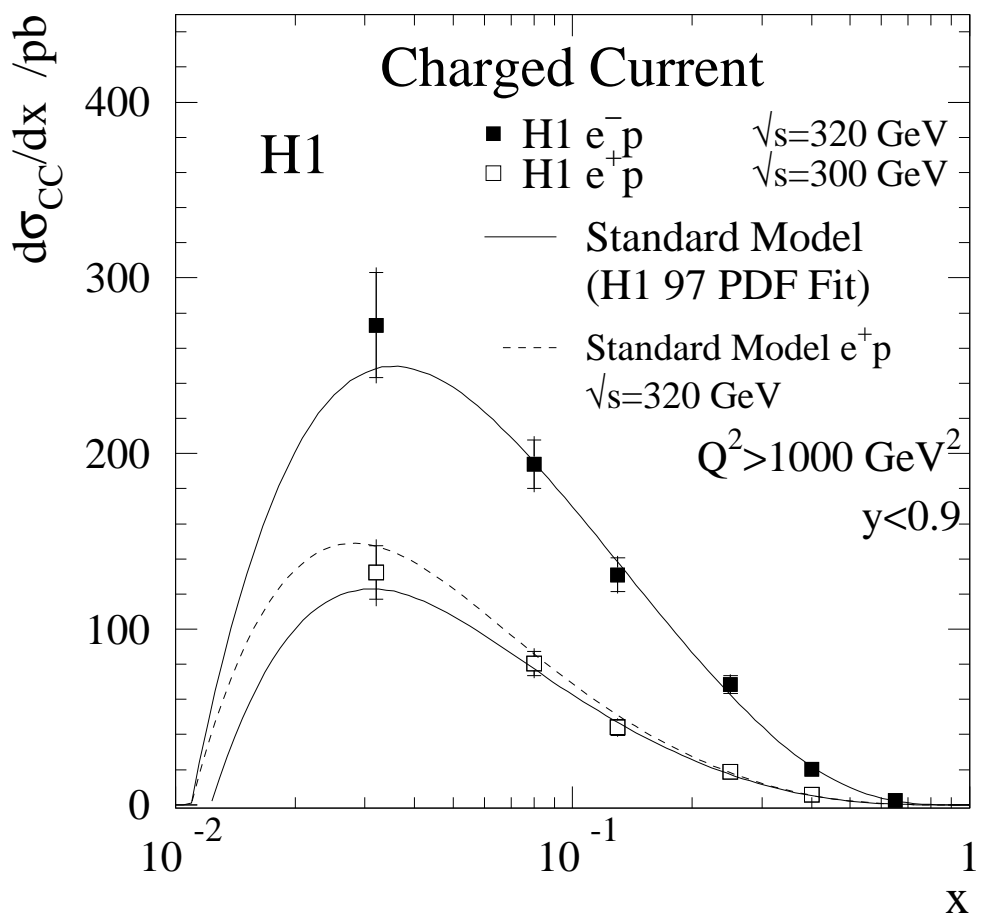

Figure 4: The $C C$ cross section $\mathrm{d} \sigma_{\mathrm{CC}} / \mathrm{d} x$ for $Q^{2}>1000 \mathrm{GeV}^{2}$ and $y<0.9$ is shown for the $\mathrm{H} 1 e^{-} p$ data (solid points) and the $\mathrm{H} 1 e^{+} p$ data [2]/(open points). The solid curves show the Standard Model expectation based on the H1 97 PDF Fit. The dashed curve shows the $e^{+} p$ cross section for an increased centre-of-mass energy. The inner error bars represent the statistical error, and the outer error bars show the total error. The normalisation uncertainties are not included in the error bars.

The $\mathrm{CC} e^{-} p$ cross section is larger than that for $e^{+} p$ scattering by a factor of two at low $x$ and a factor of four at high $x$. The difference between the cross sections is understood to be due to the different contributions of quark flavours probed by the exchanged $W^{ \pm}$bosons and the helicity structure of the $\mathrm{CC}$ interaction (see eq. 8). In the valence region at high $x$ the dominant $\mathrm{CC}$ process is the scattering off $u$ quarks for $e^{-} p$ interactions and off $d$ quarks for $e^{+} p$ interactions. The $e^{-} p$ cross section is expected to be larger (see eq. 8) since there are two $u$ valence quarks and only one $d$ valence quark in the proton. Furthermore, scattering off $d$ quarks is suppressed by a factor of $(1-y)^{2}$ compared to $u$ quarks. The effect of the increased centre-of-mass energy accounts only for a small part of the difference, and is shown by the dashed curve in fig. 4 .

\subsection{NC and CC Cross Sections $\mathrm{d} \sigma / \mathrm{dQ}^{2}$}

The NC cross section $\mathrm{d} \sigma / \mathrm{d} Q^{2}$ for $e^{-} p$ data is shown in fig. 5 for $y<0.9$ and is listed in table 4 . The cross section is corrected for the effect of the cut $y<0.63$ for $Q^{2}<890 \mathrm{GeV}^{2}$, 
the correction is also given in table 4. Also included in fig. 5 are the measurements of the $e^{+} p$ data [2, 3] and the expectations from the Standard Model. The lower plot shows the ratio of the measurement to the Standard Model expectation. The Standard Model uncertainty represents the uncertainty of the expectation due to assumptions made in the H1 97 PDF Fit, as well as the uncertainties of the experimental data entering the fit [2].

The NC data span a range of more than two orders of magnitude in $Q^{2}$ and are everywhere in good agreement with the Standard Model. At $Q^{2}<1000 \mathrm{GeV}^{2}$ there is only a small difference of $\approx 7 \%$ between the $e^{-} p$ and $e^{+} p$ measurements due to the increased centre-of-mass energy of the $e^{-}$data. For $Q^{2}>2000 \mathrm{GeV}^{2}$ the $e^{-} p$ cross section is observed to be systematically larger than the $e^{+} p$ cross section. This difference cannot be explained by the increased centre-of-mass energy, for which the expected effect on the $e^{+} p$ cross section is indicated by the dashed line. The observed asymmetry between $\mathrm{NC} e^{-} p$ and $e^{+} p$ scattering is well described by the Standard Model, where the effects of $Z^{0}$ exchange result in an enhancement of the $e^{-} p$ cross section compared to the $e^{+} p$ cross section.

The CC cross section $\mathrm{d} \sigma / \mathrm{d} Q^{2}$ for $e^{-} p$ data is shown in fig. 6 for $y<0.9$ and is listed in table 5 . The cross section is corrected for the cuts $0.03<y<0.85$ and $P_{T, h}>12 \mathrm{GeV}$. The lower plot shows the ratio of the measurements to the Standard Model expectation. The $e^{-} p$ cross section is found to be larger than the $e^{+} p$ cross section. The difference increases with $Q^{2}$ reaching a factor 10 at $Q^{2}=15000 \mathrm{GeV}^{2}$ since at higher $Q^{2}$ the average $x$ and $y$ values are larger due to the kinematic constraint $Q^{2}=s x y$. At lower $Q^{2}$, and hence lower $x$, the most important contribution arises from the sea quarks, which are approximately flavour symmetric [25] and contribute roughly equally for $e^{-} p$ and $e^{+} p$ scattering. As $Q^{2}, x$ and $y$ increase the contribution of the valence quarks becomes more important. The $\mathrm{CC}$ electron scattering cross section is in good agreement with the Standard Model expectation throughout the $Q^{2}$ range.

\subsection{NC and CC Reduced Cross Sections}

The double differential NC reduced cross section measurements are listed in table 6 and are also given in table 8 where the contribution of each of the main systematic uncertainties is listed separately. In addition table 6 gives the extracted value of the electromagnetic structure function $F_{2}$, where the correction factor, $\left(1+\Delta_{\text {all }}\right)$, was determined from the H1 97 PDF Fit. The NC reduced cross section is shown in fig. 7over the full $x$ and $Q^{2}$ range of the measurement which reaches $x=0.65$ and $Q^{2}=30000 \mathrm{GeV}^{2}$. The data exhibit a strong rise with decreasing $x$ which can be interpreted (see eq. (4) as due to the high density of low $x$ quarks in the proton. The H1 97 PDF Fit is found to give a good description of the $x, Q^{2}$ behaviour of the data, though at low $x$ the expectation has a slight tendency to be lower than the measured cross sections.

In fig. 8 the reduced cross section at high $x$ is compared with HERA measurements of $e^{+} p$ scattering and fixed target data as a function of $Q^{2}$. The $e^{-} p$ data are found to be in agreement with the $e^{+} p$ measurements for $Q^{2}<1000 \mathrm{GeV}^{2}$. The reduced cross section exhibits approximate scaling with $Q^{2}$ as is expected from the scattering of virtual photons off point-like constituents. At larger values of $Q^{2}$ the $e^{-} p$ data are generally higher than the $e^{+} p$ data, as is expected from the effects of $Z^{0}$ exchange. The data are compared with the H1 97 PDF Fit, which can be seen to give a good description of all the data up to $x=0.4$. At $x=0.65$ the fit, which in this kinematic region is mainly constrained by the BCDMS data [17], lies slightly above the H1 data. 

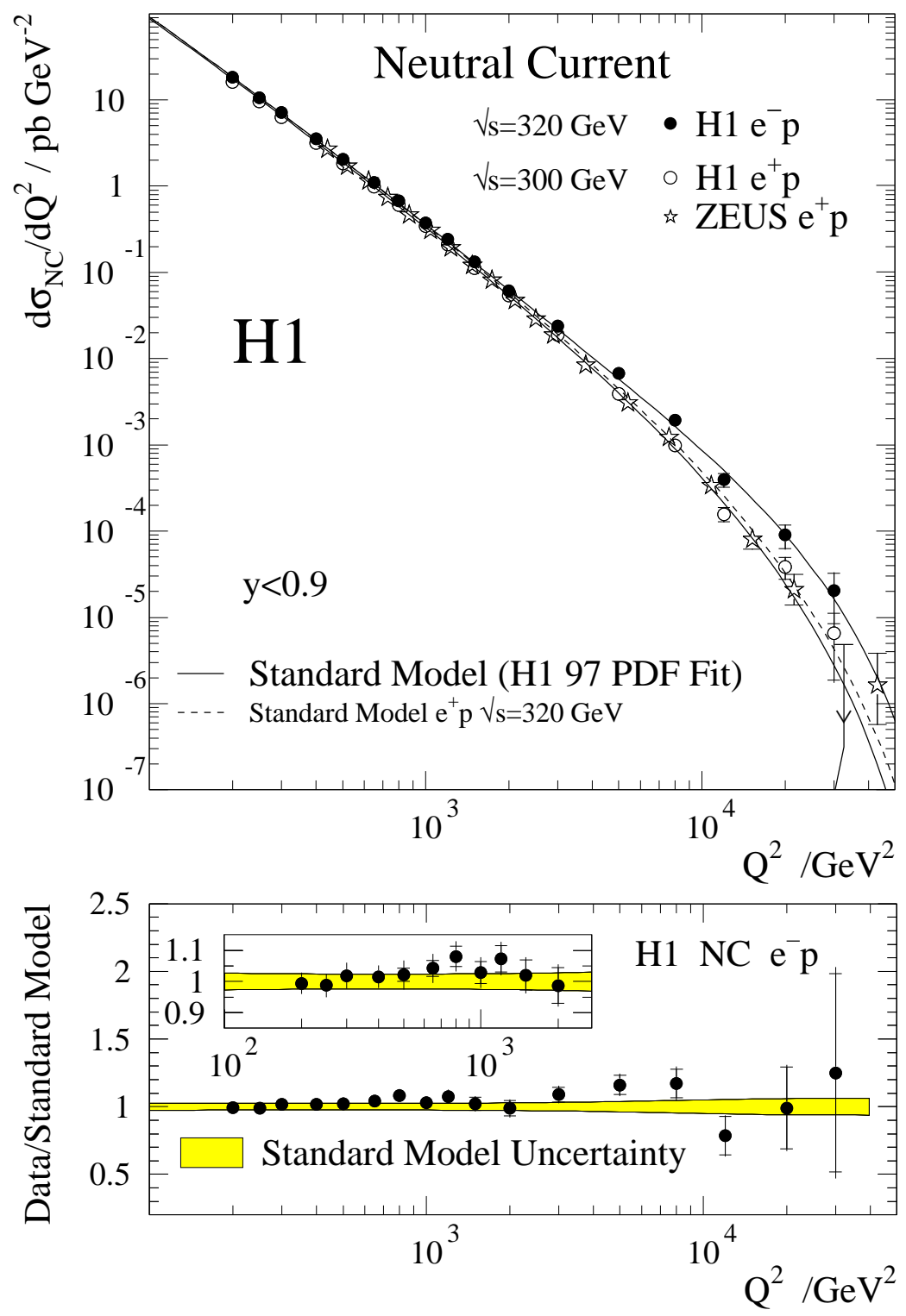

Figure 5: The $Q^{2}$ dependence of the NC cross section $\mathrm{d} \sigma_{\mathrm{NC}} / \mathrm{d} Q^{2}$ is shown for the $H 1 e^{-} p$ data (solid points) and $e^{+} p$ measurements (open points) from H1 [2] and ZEUS [3]. The data are compared with the Standard Model expectation determined from the H1 97 PDF Fit including the $\mathrm{H} 1 e^{+} p$ data. The dashed curve shows the influence of an increased centre-of-mass energy on the $e^{+} p$ cross section. The ratio of the $e^{-} p$ data to the Standard Model expectation is shown in the lower figure. The Standard Model uncertainty is shown as the shaded band. The inner error bars represent the statistical error, and the outer error bars show the total error. The normalisation uncertainties are not included in the error bars.

The level of agreement may be quantified by comparing the measurement of $\mathrm{d} \sigma / \mathrm{d} x$ at $x=0.65$ for $Q^{2}>1000 \mathrm{GeV}^{2}$ (see table (1) with the expectation from the H1 97 PDF Fit which yields a cross section of $7.05 \mathrm{pb}$. The difference is found to be less than 2.5 standard deviations. The 

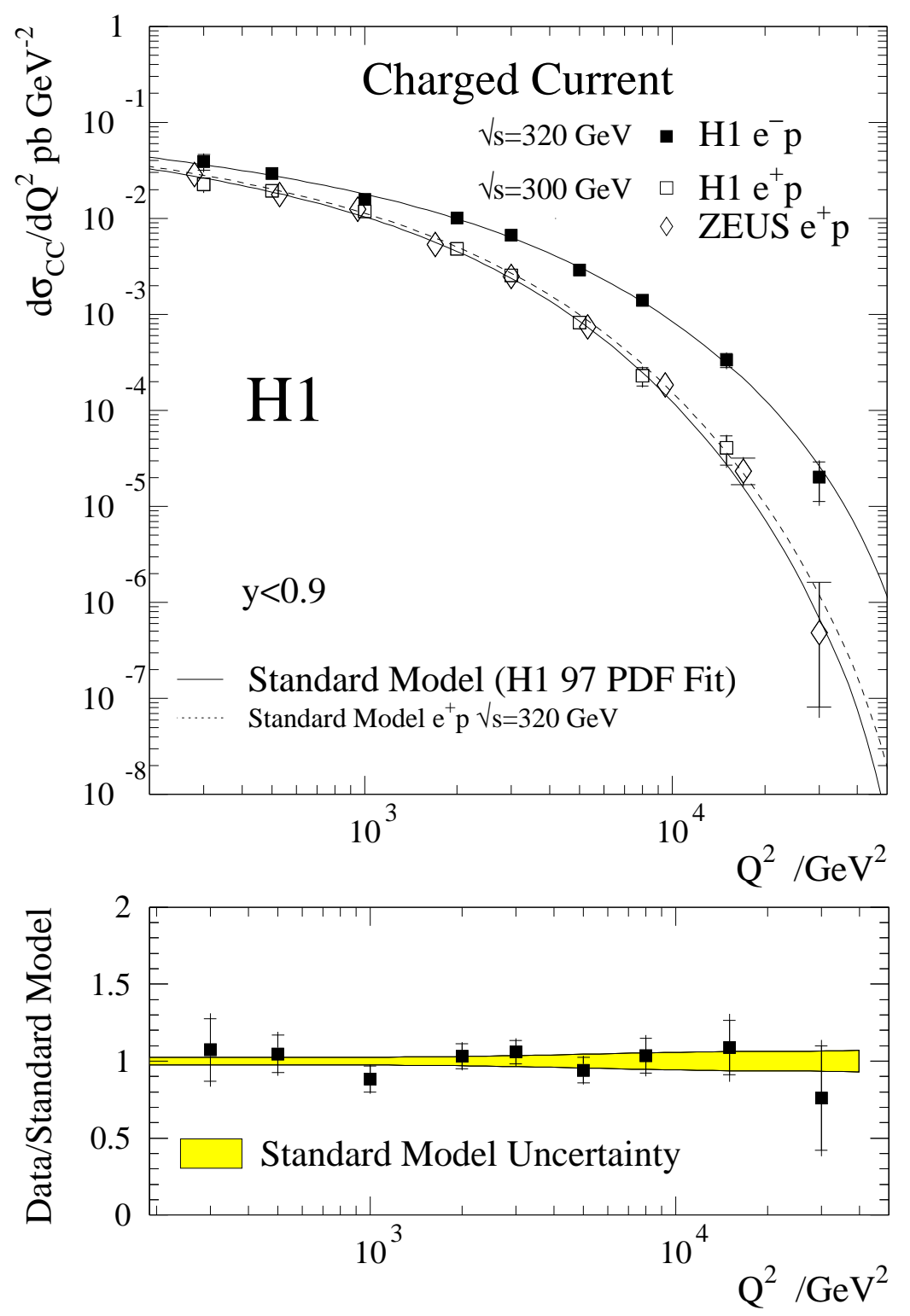

Figure 6: The $Q^{2}$ dependence of the $C C$ cross section $\mathrm{d} \sigma_{\mathrm{CC}} / \mathrm{d} Q^{2}$ is shown for the $e^{-} p$ data (solid points) and $e^{+} p$ measurements (open points) from $\mathrm{H} 1$ and ZEUS [3]. The data are compared with the Standard Model expectation determined from the H1 97 PDF Fit including the $\mathrm{H} 1 e^{+} p$ data. The dashed curve shows the influence of an increased centre-of-mass energy on the $e^{+} p$ data. The ratio of the $e^{-} p$ data to the Standard Model expectation is shown in the lower figure . The Standard Model uncertainty is shown as the shaded band. The inner error bars represent the statistical error, and the outer error bars show the total error. The normalisation uncertainties are not included in the error bars.

$\mathrm{H} 1$ data are not yet precise enough to clearly distinguish whether the effect is due to a statistical fluctuation in the $\mathrm{H} 1$ data or to systematic differences between the experiments at high $x$.

The double differential $e^{-} p \mathrm{CC}$ reduced cross section is shown in fig. 9 and compared with the 
H1 Neutral Current

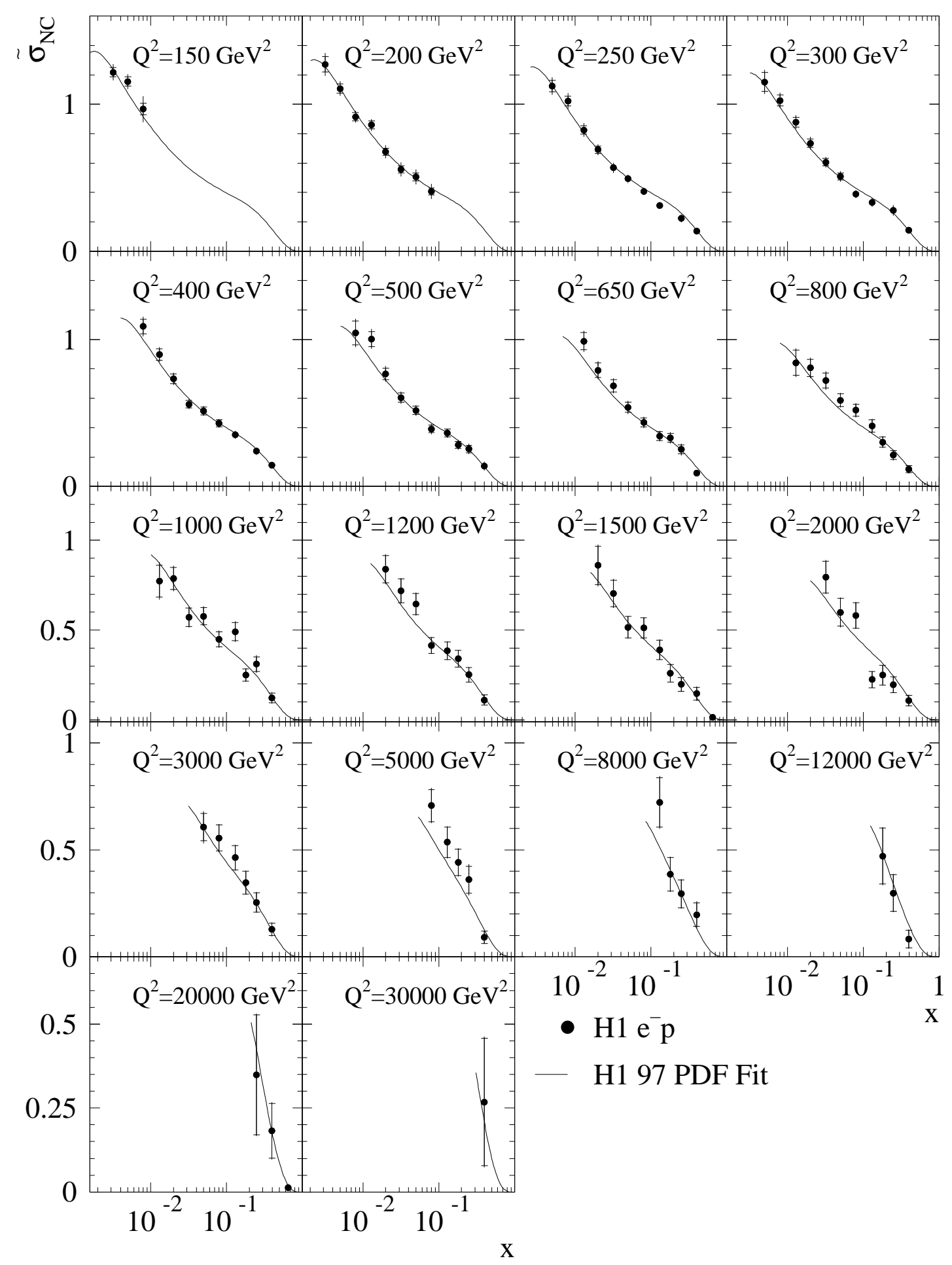

Figure 7: The $N C e^{-} p$ reduced cross section $\tilde{\sigma}_{N C}\left(x, Q^{2}\right)$ is compared with the H1 97 PDF Fit. The inner error bars represent the statistical error, and the outer error bars show the total error. The normalisation uncertainty is not included in the error bars. 


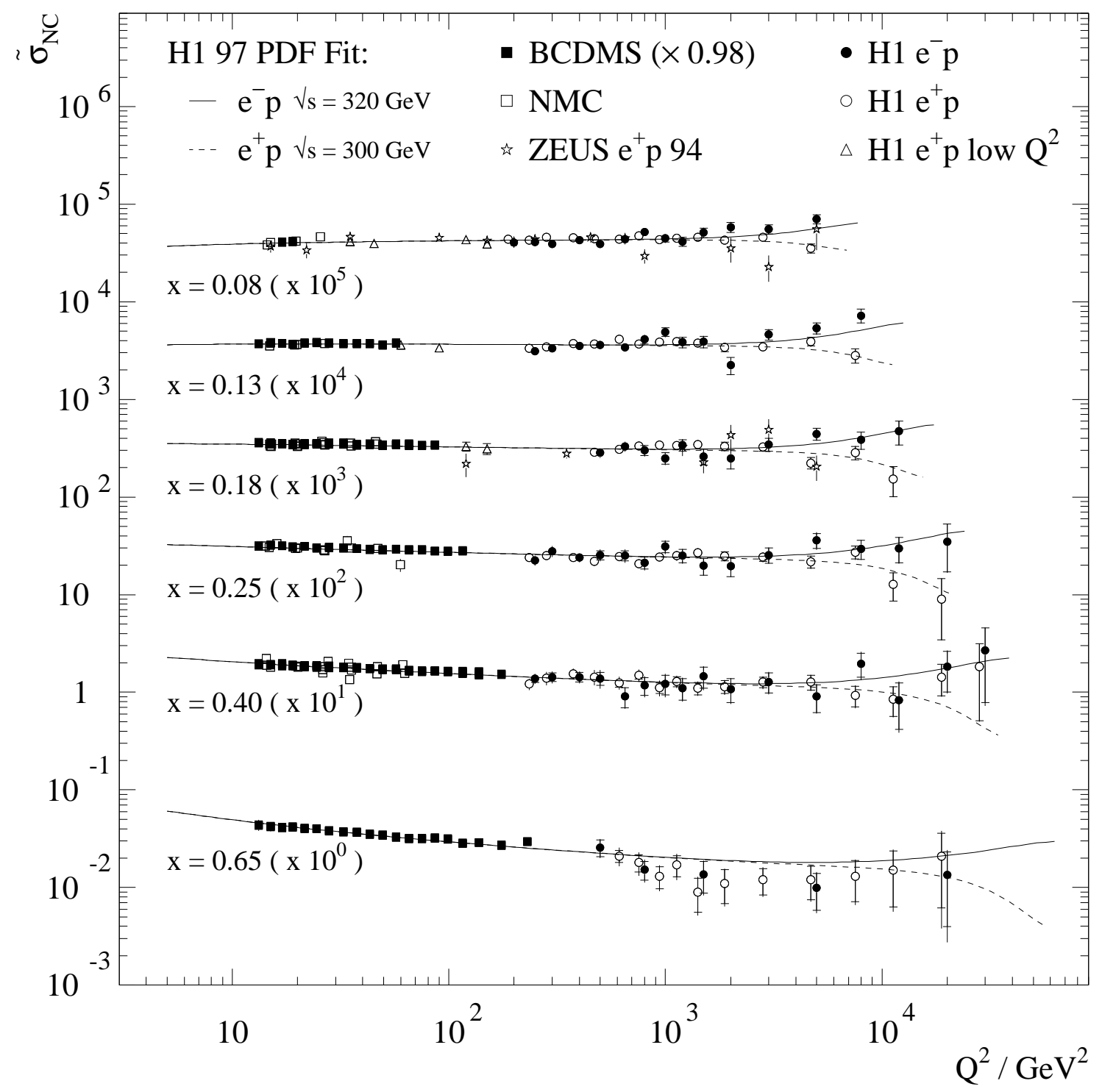

Figure 8: The NC reduced cross section $\tilde{\sigma}_{N C}\left(x, Q^{2}\right)$ is shown at high $x$ compared with the H1 97 PDF Fit. The $e^{-} p$ data with $\sqrt{s} \approx 320 \mathrm{GeV}$ (solid circles) are compared with the H1 $e^{+} p$ data at $\sqrt{s} \approx 300 \mathrm{GeV}$ (open circles), ZEUS $e^{+} p$ data [3], and fixed target data from BCDMS [17] and NMC [16]. The solid curves represent the Standard Model expectation based on the H1 97 PDF Fit. The inner error bars represent the statistical error, and the outer error bars show the total error. The normalisation uncertainties are not included in the error bars.

H1 97 PDF Fit. The data agree well with the expectation for all $x$ and $Q^{2}$. Also shown in fig. 9 is the expected contribution from the $u$ quark which dominates the $e^{-} p \mathrm{CC}$ cross section for all $x$ and $Q^{2}$. The good agreement observed between the $e^{-} p$ CC reduced cross section and the H1 97 PDF Fit indicates that the $u$ quark density of the proton is well understood across the kinematic range in the measurements. 


\section{H1 Charged Current}

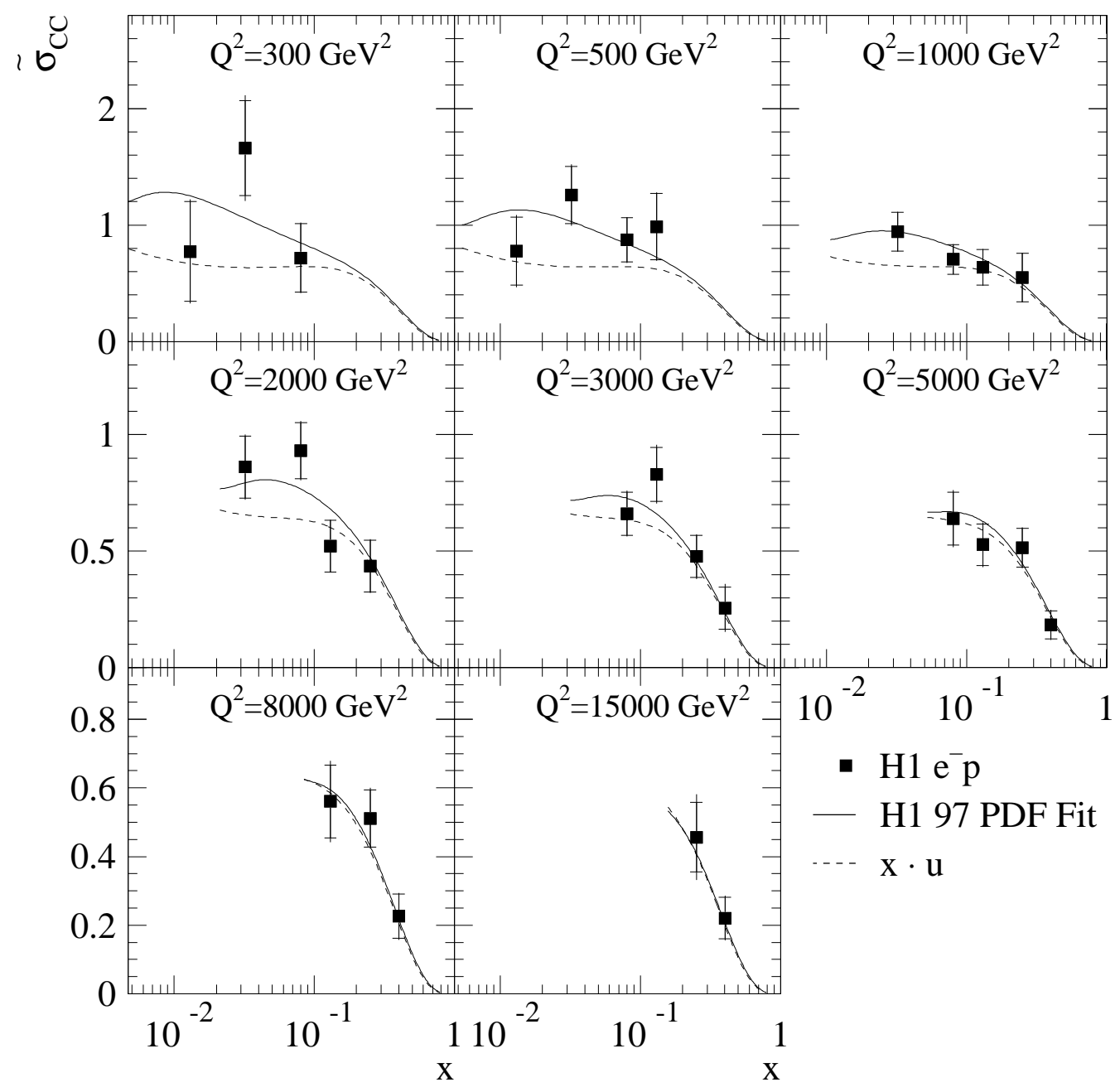

Figure 9: The $C C$ reduced cross section $\tilde{\sigma}_{C C}\left(x, Q^{2}\right)$ is shown for $e^{-} p$ scattering at $\sqrt{s} \approx$ $320 \mathrm{GeV}$ (solid points). The data are compared with the H1 97 PDF Fit. The inner error bars represent the statistical error, and the outer error bars show the total error. The normalisation uncertainty is not included in the error bars.

\subsection{Measurement of $\boldsymbol{x} \tilde{\boldsymbol{F}}_{3}$}

At large $Q^{2}$ the NC $e^{+} p$ and $e^{-} p$ cross sections differ, as expected from the effects of $Z^{0}$ exchange. The data are thus sensitive to the generalised structure function $x \tilde{F}_{3}$. Since at HERA the dominant contribution to $x \tilde{F}_{3}$ is from photon- $Z^{0}$ interference we also evaluate the structure function $x F_{3}^{\gamma Z}$, which is more closely related to the quark structure of the proton.

In order to optimise the sensitivity to $x \tilde{F}_{3}$, both the $e^{+} p$ and the $e^{-} p$ data samples with $Q^{2}$ greater than the bin boundary at $Q^{2}=1125 \mathrm{GeV}^{2}$ are rebinned into three $Q^{2}$ bins with centres $Q^{2}=1500 \mathrm{GeV}^{2}, 5000 \mathrm{GeV}^{2}$ and $12000 \mathrm{GeV}^{2}$. The reduced cross section measured in these bins is shown in fig.10(a), where the $e^{-} p$ reduced cross section is seen to be significantly higher 
than the $e^{+} p$ reduced cross section in many of the $\left(x, Q^{2}\right)$ bins shown. The structure function $x \tilde{F}_{3}$ is then evaluated using the equation

$$
\tilde{\sigma}_{N C}^{-}-\tilde{\sigma}_{N C}^{+}=x \tilde{F}_{3}\left[\frac{Y_{-920}}{Y_{+920}}+\frac{Y_{-820}}{Y_{+820}}\right]-\tilde{F}_{L}\left[\frac{y_{920}^{2}}{Y_{+920}}-\frac{y_{820}^{2}}{Y_{+820}}\right],
$$

where $y_{920}$ and $y_{820}$ are the inelasticities, and $Y_{ \pm 920}$ and $Y_{ \pm 820}$ are the helicity functions (see section (2) evaluated for fixed $x$ and $Q^{2}$ for the given proton beam energy $920 \mathrm{GeV}$ and $820 \mathrm{GeV}$. The contribution of $\tilde{F}_{L}$ was estimated from the QCD fit and is found to be $\simeq 10 \%$ at the lowest $x$ and negligible elsewhere. The resulting generalised structure function $x \tilde{F}_{3}$, shown in fig. 10 (b) as a function of $x$ is expected to rise with $Q^{2}$ for fixed $x$ due to the $Z^{0}$ propagator factor (see eq. 3). At high $x$ and low $Q^{2}$ the data are insensitive to $x \tilde{F}_{3}$, and therefore the corresponding points are removed.

The structure function $x F_{3}^{\gamma Z}$ is evaluated by dividing $x \tilde{F}_{3}$ by the factor $-a_{e} \kappa_{w} Q^{2} /\left(Q^{2}+M_{Z}^{2}\right)$. The contribution of $x F_{3}^{Z}$ is estimated to be less than $3 \%$ at the highest $Q^{2}$ and so is neglected. The measurement of $x F_{3}^{\gamma Z}$ is shown in fig. 10 (c) as a function of $x$ for three values of $Q^{2}$. The change of $x F_{3}^{\gamma Z}$ at fixed $x$ over the measured $Q^{2}$ range is expected to be very small, because it arises only from QCD scaling violations for a non-singlet structure function. It is thus reasonable to directly compare $x F_{3}^{\gamma Z}$ at the different $Q^{2}$ values.

The measurement of $x F_{3}^{\gamma Z}$ is the first at high $Q^{2}$ and also extends to lower $x$ than previous measurements. It has the advantage compared to fixed target determinations [26] in that the target is a proton, and therefore there are no corrections for nuclear effects. It should be noted that due to the quark charges and couplings (see eq. 5) $x F_{3}^{\gamma Z}$ measured in $e p$ interactions is not the same quantity as $x F_{3}^{\nu}$ as measured in $\nu N$ interactions.

The results in fig. [10(c) are consistent with zero at large $x$, rising to $\sim 0.7$ at $x \simeq 0.1$. At lower $x$ the data are consistent with expectation albeit with large errors. These observations are in agreement with the expectations from QCD in which $x F_{3}^{\gamma Z}$ is dependent on the difference between quark and anti-quark densities (see eq. 5) and is therefore primarily sensitive to the valence quark distributions. The QCD expectation for $x F_{3}^{\gamma Z}$, which is also shown in fig. 10 (c), is seen to rise to a maximum at $x \approx 0.1$. The data are found to be in agreement with the expectation at $x \gtrsim 0.2$, but lie above at lower $x$ values. In order to quantify the level of agreement of the data and the expectation the sum rule

$$
\int_{0}^{1} F_{3}^{\gamma Z} \mathrm{~d} x=2 e_{u} a_{u} N_{u}+2 e_{d} a_{d} N_{d}=\frac{5}{3} \cdot \mathcal{O}\left(1-\alpha_{s} / \pi\right)
$$

is determined [27] by analogy with the Gross Llewellyn-Smith sum rule [28] for neutrino scattering which has been found to be valid [29]. The sum rule in eq. 14 follows from eq. 5 by replacing the differences between the quark and anti-quark distributions by the valence distributions which, when integrated yield $N_{u}$ and $N_{d}$, the numbers of $u$ and $d$ valence quarks, respectively. The term $\mathcal{O}\left(1-\alpha_{s} / \pi\right)$ represents the QCD radiative corrections [30]. The H1 data at fixed $x$ are averaged by taking weighted means, and integrated yielding

$$
\int_{0.02}^{0.65} F_{3}^{\gamma Z} \mathrm{~d} x=1.88 \pm 0.35 \text { (stat.) } \pm 0.27 \text { (syst.). }
$$




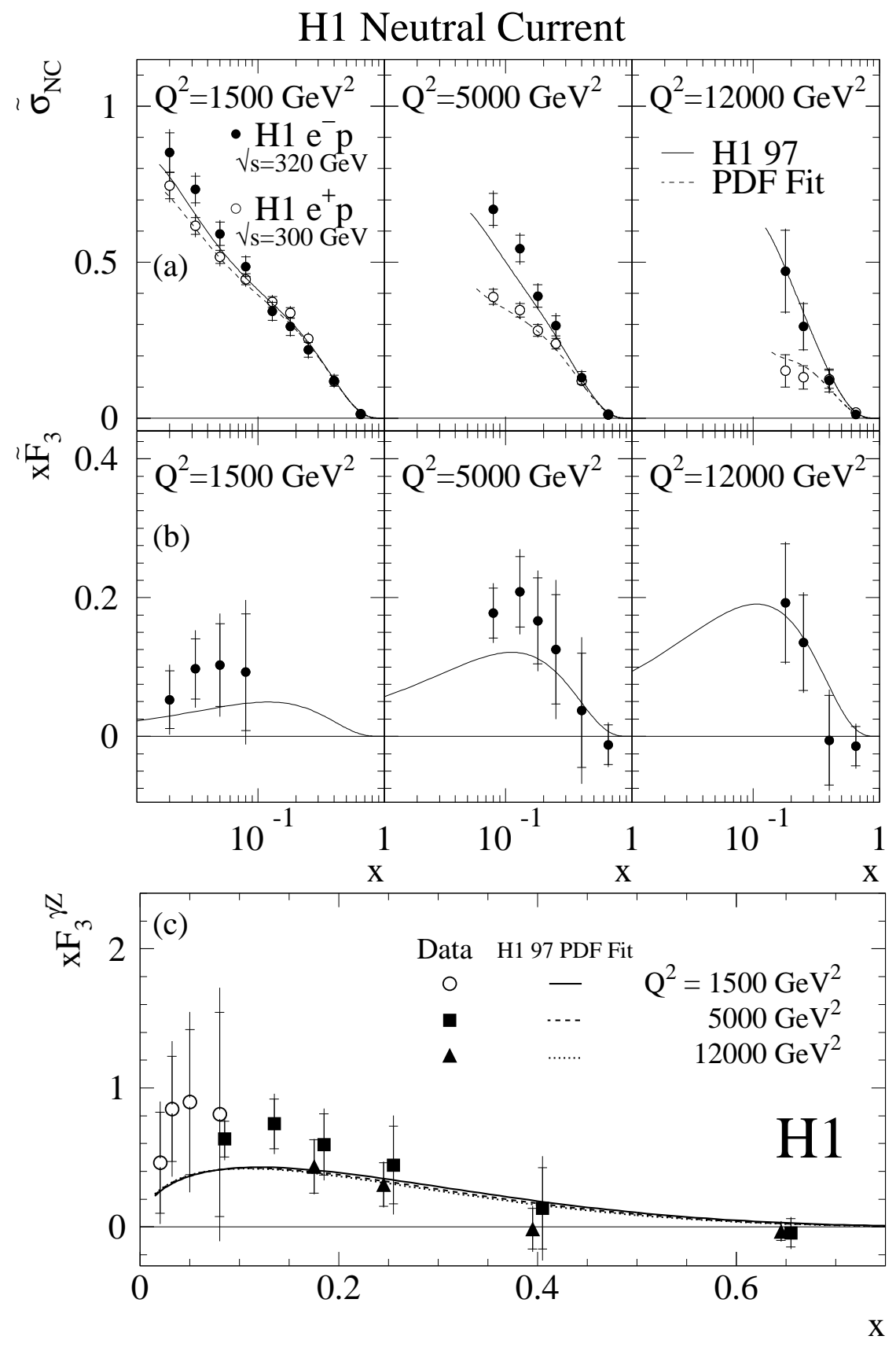

Figure 10: The NC reduced cross section $\tilde{\sigma}_{N C}\left(x, Q^{2}\right)$ is compared with the H1 97 PDF Fit for three different $Q^{2}$ values (a). The $e^{-} p$ data with $\sqrt{s} \approx 320 \mathrm{GeV}$ (solid points) are compared with the $\mathrm{H} 1 e^{+} p$ data at $\sqrt{s} \approx 300 \mathrm{GeV}$ (open points). The structure function $x \tilde{F}_{3}$ is compared with the H1 97 PDF Fit (b). The structure function $x F_{3}^{\gamma Z}$ is compared with the H1 97 PDF Fit (c). In all figures the inner error bars represent the statistical error, and the outer error bars show the total error. The normalisation uncertainties of the $e^{-} p$ and $e^{+} p$ data sets are included in the systematic errors. 
The integral obtained from the H1 97 PDF Fit gives $\int_{0.02}^{0.65} F_{3}^{\gamma Z} d x=1.11$ and when integrated over the full range in $x$ is found $/$ to be $\int_{0}^{1} F_{3}^{\gamma Z} d x=1.59$. The data and expectation are found to agree within less than two standard deviations.

\subsection{Measurement of the Total CC Cross Section and $M_{W}$}

The total CC cross section has been measured in the region $Q^{2}>1000 \mathrm{GeV}^{2}$ and $0.03<y<$ 0.85. A small correction factor for the $y$ cuts is applied ${ }^{3}$ and the cross section for the range $Q^{2}>1000 \mathrm{GeV}^{2}$ and $y<0.9$ is

$$
\sigma_{C C}^{\text {tot }}\left(e^{-} p\right)=43.08 \pm 1.84(\text { stat. }) \pm 1.74 \text { (syst.) } \mathrm{pb}
$$

where the $1.8 \%$ normalisation uncertainty is included in the systematic error. This is consistent with the expectation from the H1 97 PDF Fit where $\sigma_{C C}^{t o t}\left(e^{-} p\right)=42.70 \pm 1.65 \mathrm{pb}$.

Within the Standard Model CC interactions are mediated by the $t$-channel exchange of a $W$ boson, and therefore, are sensitive to the $W$ mass in the space-like regime. Recent determinations of the virtual $W$ mass from high $Q^{2}$ HERA data based on $e^{+} p$ scattering have been published [2, 3]. Due to the enhanced CC cross section for $e^{-} p$ compared to $e^{+} p$ scattering the new data presented here allow an improved measurement of the $W$ mass. The fit procedure is defined in [2] and uses PDFs from the H1 Low $Q^{2}$ QCD Fit [2] performed on data from NMC and BCDMS, and low $Q^{2} F_{2}$ data from $\mathrm{H} 1$ [31]. No data for $Q^{2}>100 \mathrm{GeV}^{2}$ are used in this QCD fit. The propagator mass $M_{W}$ of eq. 6 is fitted to the double differential CC cross section data, yields a $\chi^{2}$ per degree of freedom of $30.4 /(29-1)=1.09$ and a $W$ mass of

$$
M_{W}=79.9 \pm 2.2 \text { (stat.) } \pm 0.9 \text { (syst.) } \pm 2.1 \text { (theo.) } \mathrm{GeV} .
$$

The Standard Model uncertainty (theory) is determined by varying the assumptions for the Low $Q^{2}$ QCD Fit and is detailed in [2]. The weak corrections are taken into account using the HECTOR program, but are found to have negligible influence on the results. Despite a smaller luminosity than for $e^{+} p$, the larger cross section of the $e^{-} p$ data yield a more precise measurement of the $W$ mass.

\section{Summary}

Cross sections in $\mathrm{NC}$ and $\mathrm{CC}$ interactions have been measured for $e^{-} p$ scattering at a centreof-mass energy of $\sqrt{s} \approx 320 \mathrm{GeV}$ which complement earlier $e^{+} p$ measurements [2]. Standard Model expectations for deep inelastic scattering derived from the H1 97 PDF Fit to NMC, $\mathrm{BCDMS}$ and $\mathrm{H} 1 e^{+} p$ data provide a good description of the measured $e^{-} p$ cross sections thus corroborating the universality of the underlying theory.

\footnotetext{
${ }^{2}$ Note that the conditions $N_{u}=2$ and $N_{d}=1$ were constraints of the H1 97 PDF Fit.

${ }^{3}$ The factor is determined to be 1.04 from the H1 97 PDF Fit.
} 
The double differential CC reduced cross section is presented for the range $300 \leq Q^{2} \leq 15000$ $\mathrm{GeV}^{2}$, and $0.013 \leq x \leq 0.4$. The $Q^{2}$ dependence of the $\mathrm{CC}$ cross section is used to measure the $W$ propagator mass in the space-like regime. The value obtained of $M_{W}=79.9 \pm 3.2 \mathrm{GeV}$ is in good agreement with direct measurements in the time-like domain from LEP [32] and the TEVATRON [33] thus supporting the overall consistency of the Standard Model description.

Detailed comparisons of the $e^{-} p$ cross sections with the $\mathrm{H} 1$ measurements of $\mathrm{NC}$ and $\mathrm{CC} e^{+} p$ cross sections are made. The NC $e^{-} p$ measurement of $\mathrm{d} \sigma / \mathrm{d} Q^{2}$ shows a clear increase with respect to $e^{+} p$ scattering cross sections at high $Q^{2}$, consistent with the expectation of the contribution of $Z^{0}$ exchange. At $Q^{2}>10000 \mathrm{GeV}^{2}$ the cross section is found to be approximately four times larger than for $e^{+} p$ scattering. The $\mathrm{CC}$ cross section at high $Q^{2}$ is observed to be larger for electron scattering than for positron scattering by up to a factor of ten. The major part is due to different quark flavours and helicities entering the $e^{-} p$ and $e^{+} p$ cross sections. The influence of the larger centre-of-mass energy is responsible for only a small part of the increase in the $\mathrm{NC}$ and $\mathrm{CC}$ cross sections.

The double differential NC reduced cross sections are measured in the range $150 \leq Q^{2} \leq$ $30000 \mathrm{GeV}^{2}$, and $0.002 \leq x \leq 0.65$. The data agree well with measurements for $e^{+} p$ scattering for $Q^{2}<1000 \mathrm{GeV}^{2}$. At higher $Q^{2}$ the expected difference of the cross sections due to $Z^{0}$ exchange is observed and the generalised structure function $x \tilde{F}_{3}$ is measured in the range $0.02<x<0.65$ and $1500 \leq Q^{2} \leq 12000 \mathrm{GeV}^{2}$. This structure function is dominated by the interference of $\gamma$ and $Z^{0}$ which constitutes a probe of the valence quark structure of the proton. The structure function, $F_{3}^{\gamma Z}$, is explicitly derived from the measurement. The integral $\int_{0.02}^{0.65} F_{3}^{\gamma Z} \mathrm{~d} x$ is evaluated and found to be consistent with expectation within experimental errors.

\section{Acknowledgements}

We are grateful to the HERA machine group whose outstanding efforts have made and continue to make this experiment possible. We thank the engineers and technicians for their work in constructing and now maintaining the $\mathrm{H} 1$ detector, our funding agencies for financial support, the DESY technical staff for continual assistance, and the DESY directorate for the hospitality which they extend to the non-DESY members of the collaboration.

\section{References}

[1] H1 Collab., T. Ahmed et al., Phys. Lett. B324 (1994) 241;

ZEUS Collab., M. Derrick et al., Phys. Rev. Lett. 75 (1995) 1006;

H1 Collab., S. Aid et al., Z. Phys. C67 (1995) 565;

H1 Collab., S. Aid et al., Phys. Lett. B379 (1996) 319;

ZEUS Collab., M. Derrick et al., Z. Phys. C72 (1996) 47.

[2] H1 Collab., C. Adloff et al., Eur. Phys. J. C13 (2000) 609.

[3] ZEUS Collab., J. Breitweg et al., Eur. Phys. J. C11 (1999) 427;

ZEUS Collab., J. Breitweg et al., Eur. Phys. J. C12 (2000) 411. 
[4] H. Spiesberger et al., Proceedings of the Workshop "Physics at HERA", vol. 2, eds. W. Buchmüller, G. Ingelman, DESY (1992) 798.

[5] M. Klein and T. Riemann, Z. Phys. C24 (1984) 151.

[6] Review of Particle Properties, D. E. Groom et al., Eur. Phys. J. C15 (2000) 1.

[7] A. Kwiatkowski, H. Spiesberger and H.-J. Möhring, Comp. Phys. Comm. 69 (1992) 155.

[8] G.A. Schuler and H. Spiesberger, Proceedings of the Workshop "Physics at HERA", vol. 3, eds. W. Buchmüller, G. Ingelman, DESY (1992) 1419.

[9] A. Arbuzov et al., Comp. Phys. Comm. 94 (1996) 128.

[10] H1 Collab., I. Abt et al., Nucl. Instr. Meth. A386 (1997) 310 and 348.

[11] H1 SPACAL Group, R.-D.Appuhn et al., Nucl. Instr. Meth. A386 (1997) 397.

[12] R. Brun et al., GEANT3 User's Guide, CERN-DD/EE-84-1 (1987).

[13] G. Ingelman, Proceedings of the Workshop "Physics at HERA", vol. 3 eds. W. Buchmüller, G. Ingelman, DESY (1992) 1366.

[14] L. Lönnblad, Comp. Phys. Comm. 71 (1992) 15.

[15] T. Sjöstrand and M. Bengtsson, Comp. Phys. Comm. 43 (1987) 367.

[16] NMC Collab., M. Arneodo et al., Phys. Lett. B364 (1995) 107.

[17] BCDMS Collab., A.C. Benvenuti et al., Phys. Lett. B223 (1989) 485.

[18] T. Sjöstrand, Comp. Phys. Comm. 82 (1994) 74.

[19] M. Glück, E. Reya and A. Vogt, Phys. Rev. D46 (1992) 1973.

[20] U. Bassler and G. Bernardi, Nucl. Instr. Meth. A361 (1995) 197;

U. Bassler and G. Bernardi, Nucl. Instr. Meth. A426 (1999) 583.

[21] A. Blondel and F. Jacquet, Proceedings of the Study of an ep Facility for Europe, ed. U. Amaldi, DESY 79/48 (1979) 391.

[22] B. Reisert, Ph.D. Thesis, Max-Planck-Institut für Physik, Munich, MPI-PhE/2000-26, in preparation.

[23] B. Heinemann, Ph.D. Thesis, Hamburg University, 1999, DESY-THESIS-1999-046.

[24] Z. Zhang, Habilitation Thesis, LAL, 2000, LAL 00-57.

[25] E866/NuSea Collaboration, E.A. Hawker et al., Phys. Rev. Lett. 80 (1998) 3715.

[26] CDHSW Collab. P. Berge et al., Z. Phys. C49 (1991) 187;

WA25 Collab. D. Allasia et al., Z. Phys. C28 (1985) 321;

WA59 Collab. K. Varvell et al., Z. Phys. C36 (1987) 1;

CCFR Collab. W.G. Seligman et al., Phys. Rev. Lett. 79 (1997) 1213. 
[27] E. Rizvi and T. Sloan, $x F_{3}^{\gamma Z}$ in Charged Current Scattering, DESY report DESY 00-188, December 2000.

[28] D.J. Gross and C.H. Llewellyn Smith, Nucl. Phys. B14 (1969) 337.

[29] CCFR Collab., J.H. Kim et al., Phys. Rev. Lett. 81 (1998) 3595.

[30] S.A. Larin and J.A.M. Vermaseren, Phys. Lett. B259 (1991) 345.

[31] H1 Collab., S. Aid et al., Nucl. Phys. B 470 (1996) 3.

[32] ALEPH Collab., R. Barate et al., Phys. Lett. B422 (1998) 384;

DELPHI Collab., P. Abreu et al., Eur. Phys. J. C2 (1998) 581;

L3 Collab., M. Acciarri et al., Phys. Lett. B413 (1997) 176;

OPAL Collab., K. Ackerstaff et al., Eur. Phys. J. C1 (1998) 395.

[33] CDF Collab., F. Abe et al., Phys. Rev. Lett. 75 (1995) 11;

D $\emptyset$ Collab., B. Abbott et al., Phys. Rev. Lett. 80 (1998) 3008. 


\begin{tabular}{|c|c||r|r|r|r|}
\hline$x$ & $\begin{array}{c}\mathrm{d} \sigma_{N C} / \mathrm{d} x(\mathrm{pb}) \\
Q^{2}>1000 \mathrm{GeV}^{2}, \mathrm{y}<0.9\end{array}$ & $\begin{array}{r}\delta_{\text {sta }} \\
(\%)\end{array}$ & $\begin{array}{r}\delta_{\text {unc }} \\
(\%)\end{array}$ & $\begin{array}{r}\delta_{\text {cor }} \\
(\%)\end{array}$ & $\begin{array}{r}\delta_{\text {tot }} \\
(\%)\end{array}$ \\
\hline 0.013 & $0.126 \cdot 10^{4}$ & 13.6 & 5.7 & 4.6 & 15.5 \\
0.020 & $0.276 \cdot 10^{4}$ & 6.0 & 3.1 & 1.9 & 7.0 \\
0.032 & $0.246 \cdot 10^{4}$ & 4.9 & 2.9 & 1.0 & 5.8 \\
0.050 & $0.172 \cdot 10^{4}$ & 4.8 & 3.0 & 1.1 & 5.8 \\
0.080 & $0.115 \cdot 10^{4}$ & 4.5 & 3.1 & 1.1 & 5.6 \\
0.130 & $0.636 \cdot 10^{3}$ & 5.4 & 3.5 & 1.0 & 6.5 \\
0.180 & $0.383 \cdot 10^{3}$ & 6.2 & 3.3 & 1.3 & 7.1 \\
0.250 & $0.221 \cdot 10^{3}$ & 6.9 & 6.0 & 2.8 & 9.6 \\
0.400 & $0.693 \cdot 10^{2}$ & 9.8 & 9.1 & 4.2 & 14.0 \\
0.650 & $0.421 \cdot 10^{1}$ & 24.3 & 14.8 & 6.3 & 29.2 \\
\hline
\end{tabular}

Table 1: The NC cross section $\mathrm{d} \sigma_{N C} / \mathrm{d} x$ measured for $y<0.9$ and $Q^{2}>1000 \mathrm{GeV}^{2}$. The statistical $\left(\delta_{\text {sta }}\right)$, uncorrelated systematic $\left(\delta_{u n c}\right)$, correlated systematic $\left(\delta_{\text {cor }}\right)$, and total $\left(\delta_{\text {tot }}\right)$ errors are also given. The normalisation uncertainty of $1.8 \%$ is not included in the errors.

\begin{tabular}{|c|c||r|r|r|r|}
\hline$x$ & $\begin{array}{c}\mathrm{d} \sigma_{N C} / \mathrm{d} x(\mathrm{pb}) \\
Q^{2}>10000 \mathrm{GeV}^{2}, \mathrm{y}<0.9\end{array}$ & $\begin{array}{r}\delta_{\text {sta }} \\
(\%)\end{array}$ & $\begin{array}{r}\delta_{\text {unc }} \\
(\%)\end{array}$ & $\begin{array}{r}\delta_{\text {cor }} \\
(\%)\end{array}$ & $\begin{array}{r}\delta_{\text {tot }} \\
(\%)\end{array}$ \\
\hline 0.130 & $0.473 \cdot 10^{1}$ & 82.9 & 19.3 & 5.2 & 85.3 \\
0.180 & $0.156 \cdot 10^{2}$ & 26.9 & 6.5 & 3.1 & 27.9 \\
0.250 & $0.110 \cdot 10^{2}$ & 25.2 & 7.2 & 2.1 & 26.3 \\
0.400 & $0.354 \cdot 10^{1}$ & 30.2 & 13.7 & 3.5 & 33.3 \\
0.650 & $0.024 \cdot 10^{1}$ & 70.7 & 33.9 & 13.4 & 79.6 \\
\hline
\end{tabular}

Table 2: The NC cross section $\mathrm{d} \sigma_{N C} / \mathrm{d} x$ measured for $y<0.9$ and $Q^{2}>10000 \mathrm{GeV}^{2}$. The statistical $\left(\delta_{\text {sta }}\right)$, uncorrelated systematic $\left(\delta_{\text {unc }}\right)$, correlated systematic $\left(\delta_{\text {cor }}\right)$, and total $\left(\delta_{\text {tot }}\right)$ errors are also given. The normalisation uncertainty of $1.8 \%$ is not included in the errors. 


\begin{tabular}{|c|c||c|c|c|c|c|}
\hline$x$ & $\begin{array}{c}\mathrm{d} \sigma_{C C} / \mathrm{d} x(\mathrm{pb}) \\
Q^{2}>1000 \mathrm{GeV}^{2}, y<0.9\end{array}$ & $k_{\text {cor }}$ & $\begin{array}{c}\delta_{\text {sta }} \\
(\%)\end{array}$ & $\begin{array}{c}\delta_{\text {unc }} \\
(\%)\end{array}$ & $\begin{array}{c}\delta_{\text {cor }} \\
(\%)\end{array}$ & $\begin{array}{c}\delta_{\text {tot }} \\
(\%)\end{array}$ \\
\hline 0.032 & $0.273 \cdot 10^{3}$ & 1.064 & 11.0 & 4.6 & 2.4 & 12.1 \\
0.080 & $0.194 \cdot 10^{3}$ & 1.033 & 7.1 & 3.9 & 1.2 & 8.2 \\
0.130 & $0.131 \cdot 10^{3}$ & 1.023 & 7.3 & 3.4 & 1.0 & 8.1 \\
0.250 & $0.686 \cdot 10^{2}$ & 1.014 & 7.4 & 3.9 & 1.1 & 8.4 \\
0.400 & $0.201 \cdot 10^{2}$ & 1.051 & 14.1 & 11.0 & 4.8 & 18.5 \\
0.650 & $0.264 \cdot 10^{1}$ & 1.197 & 49.8 & 21.1 & 16.9 & 56.7 \\
\hline
\end{tabular}

Table 3: The CC cross-section $\mathrm{d} \sigma_{C C} / \mathrm{d} x$ measured for $Q^{2}>1000 \mathrm{GeV}^{2}$ and $0.03<y<0.85$ after correction $\left(k_{\mathrm{cor}}\right)$ to $y<0.9$. The statistical $\left(\delta_{\text {sta }}\right)$, uncorrelated systematic $\left(\delta_{\text {unc }}\right)$, correlated systematic $\left(\delta_{\text {cor }}\right)$, and total $\left(\delta_{\text {tot }}\right)$ errors are also given. The normalisation uncertainty of $1.8 \%$ is not included in the errors.

\begin{tabular}{|r|l|l||r|r|r|r|}
\hline $\begin{array}{r}Q^{2} \\
\left(\mathrm{GeV}^{2}\right)\end{array}$ & $\begin{array}{l}\mathrm{d} \sigma_{N C} / \mathrm{d}^{2} \\
\left(\mathrm{pb} / \mathrm{GeV}^{2}\right) \\
y<0.9\end{array}$ & $k_{\text {cor }}$ & $\begin{array}{r}\delta_{\text {sta }} \\
(\%)\end{array}$ & $\begin{array}{r}\delta_{\text {unc }} \\
(\%)\end{array}$ & $\begin{array}{r}\delta_{\text {cor }} \\
(\%)\end{array}$ & $\begin{array}{r}\delta_{\text {tot }} \\
(\%)\end{array}$ \\
\hline 200 & $1.822 \cdot 10^{1}$ & 1.078 & 1.3 & 3.0 & 1.1 & 3.4 \\
250 & $1.067 \cdot 10^{1}$ & 1.075 & 1.3 & 3.2 & 1.7 & 3.9 \\
300 & $7.109 \cdot 10^{0}$ & 1.070 & 1.5 & 3.4 & 1.7 & 4.1 \\
400 & $3.536 \cdot 10^{0}$ & 1.060 & 1.7 & 2.8 & 1.3 & 3.6 \\
500 & $2.062 \cdot 10^{0}$ & 1.048 & 2.1 & 3.5 & 2.2 & 4.6 \\
650 & $1.103 \cdot 10^{0}$ & 1.031 & 2.5 & 3.3 & 1.7 & 4.5 \\
800 & $0.683 \cdot 10^{0}$ & 1.013 & 3.0 & 3.2 & 1.1 & 4.5 \\
1000 & $0.372 \cdot 10^{0}$ & 1.000 & 3.4 & 3.1 & 1.3 & 4.8 \\
1200 & $0.245 \cdot 10^{0}$ & 1.000 & 3.9 & 3.0 & 0.9 & 5.0 \\
1500 & $0.132 \cdot 10^{0}$ & 1.000 & 4.8 & 3.0 & 1.0 & 5.7 \\
2000 & $0.615 \cdot 10^{-1}$ & 1.000 & 5.8 & 3.3 & 1.7 & 6.8 \\
3000 & $0.239 \cdot 10^{-1}$ & 1.000 & 5.0 & 3.1 & 0.9 & 6.0 \\
5000 & $0.675 \cdot 10^{-2}$ & 1.000 & 6.1 & 3.5 & 0.8 & 7.1 \\
8000 & $0.191 \cdot 10^{-2}$ & 1.000 & 9.1 & 5.7 & 1.0 & 10.8 \\
12000 & $0.395 \cdot 10^{-3}$ & 1.000 & 18.2 & 6.7 & 1.3 & 19.4 \\
20000 & $0.901 \cdot 10^{-4}$ & 1.000 & 30.6 & 10.2 & 1.4 & 32.2 \\
30000 & $0.204 \cdot 10^{-4}$ & 1.000 & 58.5 & 21.8 & 3.0 & 62.5 \\
\hline
\end{tabular}

Table 4: The NC cross section $\mathrm{d} \sigma_{N C} / \mathrm{d} Q^{2}$ for $y<0.9$ after correction $\left(k_{\text {cor }}\right)$ according to the Standard Model expectation for the kinematic cuts $y<0.63$ for $Q^{2}<890 \mathrm{GeV}^{2}$. The statistical $\left(\delta_{\text {sta }}\right)$, uncorrelated systematic $\left(\delta_{\text {unc }}\right)$, correlated systematic $\left(\delta_{c o r}\right)$, and total $\left(\delta_{\text {tot }}\right)$ errors are also given. The normalisation uncertainty of $1.8 \%$ is not included in the errors. 


\begin{tabular}{|c|c||c|c|c|c|c||c|}
\hline $\begin{array}{c}Q^{2} \\
\left(\mathrm{GeV}^{2}\right)\end{array}$ & $\begin{array}{c}\mathrm{d} \sigma_{C C} / \mathrm{d}^{2} \\
\left(\mathrm{pb} / \mathrm{GeV}^{2}\right) \\
y<0.9\end{array}$ & $k_{\text {cor }}$ & $\begin{array}{c}\delta_{\text {sta }} \\
(\%)\end{array}$ & $\begin{array}{c}\delta_{\text {unc }} \\
(\%)\end{array}$ & $\begin{array}{c}\delta_{\text {cor }} \\
(\%)\end{array}$ & $\begin{array}{c}\delta_{\text {tot }} \\
(\%)\end{array}$ & $\begin{array}{c}\delta_{\text {qed }} \\
(\%)\end{array}$ \\
\hline 300 & $0.389 \cdot 10^{-1}$ & 1.495 & 18.8 & 7.2 & 9.5 & 22.3 & 4.2 \\
500 & $0.292 \cdot 10^{-1}$ & 1.245 & 11.7 & 5.3 & 4.3 & 13.6 & 2.1 \\
1000 & $0.158 \cdot 10^{-1}$ & 1.070 & 9.7 & 4.2 & 2.0 & 10.7 & -0.6 \\
2000 & $0.102 \cdot 10^{-1}$ & 1.024 & 7.8 & 4.0 & 1.4 & 8.8 & -1.5 \\
3000 & $0.667 \cdot 10^{-2}$ & 1.026 & 7.3 & 4.2 & 0.6 & 8.4 & -0.8 \\
5000 & $0.292 \cdot 10^{-2}$ & 1.034 & 8.8 & 4.3 & 0.9 & 9.9 & -4.2 \\
8000 & $0.140 \cdot 10^{-2}$ & 1.046 & 10.8 & 6.6 & 2.5 & 12.9 & -8.0 \\
15000 & $0.336 \cdot 10^{-3}$ & 1.080 & 16.3 & 11.2 & 4.8 & 20.3 & -13.6 \\
30000 & $0.202 \cdot 10^{-4}$ & 1.183 & 44.6 & 18.7 & 10.9 & 49.6 & -20.7 \\
\hline
\end{tabular}

Table 5: The $C C$ cross-section $\mathrm{d} \sigma_{C C} / \mathrm{d} Q^{2}$ for $y<0.9$ after correction $\left(k_{\text {cor }}\right)$ according to Standard Model expectations for kinematic cuts $0.03<y<0.85$ and $P_{T, h}>12 \mathrm{GeV}$. The statistical $\left(\delta_{\text {sta }}\right)$, uncorrelated systematic $\left(\delta_{\text {unc }}\right)$, correlated systematic $\left(\delta_{\text {cor }}\right)$, and total $\left(\delta_{\text {tot }}\right)$ errors are also given. The final column gives the correction for $Q E D$ radiative effets $\delta_{C C}^{q e d}$. The normalisation uncertainty of $1.8 \%$ is not included in the errors. 


\begin{tabular}{|c|c|c|c|c|c|c|c|c|c|c|c|}
\hline $\begin{array}{r}Q^{2} \\
\left(\mathrm{GeV}^{2}\right) \\
\end{array}$ & $x$ & $y$ & $\tilde{\sigma}_{N C}$ & $\begin{array}{l}\delta_{\text {sta }} \\
(\%)\end{array}$ & $\begin{array}{l}\delta_{\text {sys }} \\
(\%)\end{array}$ & $\begin{array}{c}\delta_{t o t} \\
(\%)\end{array}$ & $F_{2}$ & $\begin{array}{l}\Delta_{\text {all }} \\
(\%) \\
\end{array}$ & $\begin{array}{l}\Delta_{F_{2}} \\
(\%)\end{array}$ & $\begin{array}{l}\Delta_{F_{3}} \\
(\%)\end{array}$ & $\begin{array}{r}\Delta_{F_{L}} \\
(\%) \\
\end{array}$ \\
\hline 150 & 0.0032 & 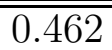 & $\bar{~} 1.218$ & $\overline{2.7}$ & $\overline{3.8}$ & $\overline{44.7}$ & $\overline{c 1.253}$ & $=2.8$ & 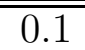 & 0.1 & $\overline{-3.0}$ \\
\hline 150 & 0.0050 & 0.295 & 1.154 & 2.8 & 3.4 & 4.4 & 1.164 & -0.8 & 0.1 & 0.1 & -1.0 \\
\hline 150 & 0.0080 & 0.185 & 0.968 & 4.1 & 8.2 & 9.1 & 0.969 & -0.2 & 0.1 & 0.0 & -0.3 \\
\hline 200 & 0.0032 & 0.615 & 1.271 & 4.1 & 4.5 & 6.1 & 1.344 & -5.4 & 0.2 & 0.1 & -5.7 \\
\hline 200 & 0.0050 & 0.394 & 1.107 & 2.8 & 3.6 & 4.6 & 1.125 & -1.6 & 0.2 & 0.1 & -1.8 \\
\hline 200 & 0.0080 & 0.246 & 0.915 & 3.0 & 3.3 & 4.5 & 0.918 & -0.4 & 0.2 & 0.1 & -0.6 \\
\hline 200 & 0.0130 & 0.152 & 0.860 & 3.2 & 3.5 & 4.7 & 0.859 & 0.0 & 0.2 & 0.1 & -0.2 \\
\hline 200 & 0.0200 & 0.099 & 0.677 & 3.8 & 5.3 & 6.5 & 0.676 & 0.2 & 0.2 & 0.1 & -0.1 \\
\hline 200 & 0.0320 & 0.062 & 0.558 & 4.5 & 7.4 & 8.6 & 0.556 & 0.2 & 0.2 & 0.1 & 0.0 \\
\hline 200 & 0.0500 & 0.039 & 0.506 & 5.2 & 8.4 & 9.9 & 0.505 & 0.2 & 0.2 & 0.1 & 0.0 \\
\hline 200 & 0.0800 & 0.025 & 0.407 & 5.9 & 10.9 & 12.4 & 0.406 & 0.2 & 0.1 & 0.0 & 0.0 \\
\hline 250 & 0.0050 & 0.492 & 1.123 & 3.5 & 4.0 & 5.3 & 1.154 & -2.7 & 0.2 & 0.1 & -3.0 \\
\hline 250 & 0.0080 & 0.308 & 1.021 & 3.2 & 4.2 & 5.3 & 1.027 & -0.6 & 0.2 & 0.1 & -0.9 \\
\hline 250 & 0.0130 & 0.189 & 0.825 & 3.4 & 4.5 & 5.7 & 0.825 & 0.0 & 0.2 & 0.1 & -0.3 \\
\hline 250 & 0.0200 & 0.123 & 0.691 & 3.5 & 4.0 & 5.4 & 0.689 & 0.2 & 0.2 & 0.1 & -0.1 \\
\hline 250 & 0.0320 & 0.077 & 0.569 & 3.8 & 4.7 & 6.1 & 0.567 & 0.3 & 0.2 & 0.1 & 0.0 \\
\hline 250 & 0.0 & & 0 . & 4.3 & 3.7 & 5.7 & & 0.3 & 0.2 & 0.1 & 0.0 \\
\hline 250 & 0.0800 & 0.031 & 0.407 & 4.7 & 3.9 & 6.1 & 0.406 & 0.3 & 0.2 & 0.1 & 0.0 \\
\hline 250 & 0.1300 & 0.019 & 0.311 & 5.3 & 5.8 & 7.8 & 0.310 & 0.2 & 0.2 & 0.1 & 0.0 \\
\hline 250 & 0.2500 & 0.010 & 0.225 & 7.5 & 9.5 & 12.1 & 0.224 & 0.2 & 0.2 & 0.0 & 0.0 \\
\hline 250 & 0.4000 & 0.006 & 0.138 & 9.3 & 7.2 & 11.8 & 0.138 & 0.2 & 0.2 & 0.0 & 0.0 \\
\hline 300 & 0.0050 & 0.591 & 1.152 & 5.6 & 4.6 & 7.2 & 1.202 & -4.1 & 0.3 & 0.2 & -4.6 \\
\hline 300 & 0.0080 & 0.369 & 1.026 & 3.6 & 3.6 & 5.1 & 1.036 & -0.9 & 0.3 & 0.2 & -1.4 \\
\hline 300 & 0.0130 & 0.227 & 0.878 & 3.8 & 3.7 & 5.3 & 0.878 & 0.0 & 0.3 & 0.2 & -0.4 \\
\hline 300 & 0.0200 & 0.148 & 0.735 & 4.0 & 4.3 & 5.9 & 0.733 & 0.3 & 0.3 & 0.1 & -0.1 \\
\hline 300 & 0.0320 & 0.092 & 0.605 & 4.2 & 4.1 & 5.8 & 0.603 & 0.3 & 0.3 & 0.1 & 0.0 \\
\hline 300 & 0.0500 & 0.059 & 0.509 & 4.5 & 5.1 & 6.8 & 0.507 & 0.4 & 0.3 & 0.1 & 0.0 \\
\hline 300 & 0.0800 & 0.037 & 0.390 & 5.2 & 4.6 & 6.9 & 0.389 & 0.3 & 0.2 & 0.1 & 0.0 \\
\hline 300 & 0.1300 & 0.023 & 0.332 & 5.4 & 7.0 & 8.8 & 0.331 & 0.3 & 0.2 & 0.1 & 0.0 \\
\hline 300 & 0.2500 & 0.012 & 0.277 & 6.9 & 10.8 & 12.8 & 0.277 & 0.3 & 0.2 & 0.0 & 0.0 \\
\hline 300 & 0.4000 & 0.007 & 0.143 & 10.3 & 9.8 & 14.2 & 0.142 & 0.2 & 0.2 & 0.0 & 0.0 \\
\hline 400 & 0.0080 & 0.492 & 1.088 & 4.5 & 4.1 & 6.1 & 1.109 & -1.9 & 0.4 & 0.3 & -2.6 \\
\hline 400 & 0.0130 & 0.303 & 0.897 & 4.3 & 3.6 & 5.6 & 0.898 & -0.1 & 0.4 & 0.3 & -0.8 \\
\hline 400 & 0.0200 & 0.197 & 0.732 & 4.5 & 3.6 & 5.8 & 0.729 & 0.4 & 0.4 & 0.3 & -0.3 \\
\hline 400 & 0.0320 & 0.123 & 0.560 & 4.8 & 3.8 & 6.1 & 0.557 & 0.5 & 0.4 & 0.2 & -0.1 \\
\hline 400 & 0.0500 & 0.079 & 0.514 & 5.0 & 3.7 & 6.3 & 0.511 & 0.5 & 0.4 & 0.2 & 0.0 \\
\hline 400 & 0.0800 & 0.049 & 0.429 & 5.5 & 4.3 & 7.0 & 0.427 & 0.5 & 0.4 & 0.2 & 0.0 \\
\hline 400 & 0.1300 & 0.030 & 0.352 & 5.6 & 5.0 & 7.5 & 0.351 & 0.5 & 0.3 & 0.1 & 0.0 \\
\hline
\end{tabular}

Table 6: The NC reduced cross section $\tilde{\sigma}_{N C}\left(x, Q^{2}\right)$ with statistical $\left(\delta_{\text {sta }}\right)$, systematic $\left(\delta_{\text {sys }}\right)$, and total $\left(\delta_{\text {tot }}\right)$ errors. The electromagnetic structure function $F_{2}$ is also given with the corrections $\Delta_{\text {all }}, \Delta_{F_{2}}, \Delta_{F_{3}}, \Delta_{F_{L}}$ as defined in eq. 10 . The normalisation uncertainty of $1.8 \%$ is not included in the errors. The table continues on the next 3 pages. 


\begin{tabular}{|c|c|c|c|c|c|c|c|c|c|c|c|}
\hline $\begin{array}{r}Q^{2} \\
\left(\mathrm{GeV}^{2}\right) \\
\end{array}$ & $x$ & $y$ & $\tilde{\sigma}_{N C}$ & $\begin{array}{l}\delta_{\text {sta }} \\
(\%) \\
\end{array}$ & $\begin{array}{l}\delta_{\text {sys }} \\
(\%)\end{array}$ & $\begin{array}{r}\delta_{t o t} \\
(\%) \\
\end{array}$ & $F_{2}$ & $\begin{array}{l}\Delta_{\text {all }} \\
(\%) \\
\end{array}$ & $\begin{array}{l}\Delta_{F_{2}} \\
(\%) \\
\end{array}$ & $\begin{array}{l}\Delta_{F_{3}} \\
(\%) \\
\end{array}$ & $\begin{array}{r}\Delta_{F_{L}} \\
(\%) \\
\end{array}$ \\
\hline 400 & 0.2500 & 0.016 & 0.240 & 7.6 & 7.4 & $\overline{10.6}$ & 0.239 & $\overline{0.4}$ & 0.3 & 0.1 & 0.0 \\
\hline 400 & 0.4000 & 0.010 & 0.143 & 10.8 & 8.4 & 13.7 & 0.143 & 0.3 & 0.3 & 0.1 & 0.0 \\
\hline 500 & 0.0080 & 0.615 & 1.044 & 7.8 & 5.1 & 9.3 & 1.080 & -3.3 & 0.5 & 0.5 & -4.3 \\
\hline 500 & 0.0130 & 0.379 & 1.003 & 5.1 & 4.5 & 6.8 & 1.006 & -0.3 & 0.5 & 0.4 & -1.2 \\
\hline 500 & 0.0200 & 0.246 & 0.765 & 5.1 & 4.8 & 7.0 & 0.761 & 0.5 & 0.5 & 0.4 & -0.4 \\
\hline 500 & 0.0320 & 0.154 & 0.604 & 5.3 & 4.5 & 7.0 & 0.600 & 0.7 & 0.5 & 0.4 & -0.1 \\
\hline 500 & 0.0500 & 0.099 & 0.517 & 5.6 & 4.0 & 6.9 & 0.513 & 0.8 & 0.5 & 0.3 & 0.0 \\
\hline 500 & 0.0800 & 0.062 & 0.392 & 6.4 & 6.5 & 9.2 & 0.389 & 0.7 & 0.5 & 0.3 & 0.0 \\
\hline 500 & 0.1300 & 0.038 & 0.363 & 7.2 & 4.9 & 8.7 & 0.361 & 0.6 & 0.4 & 0.2 & 0.0 \\
\hline 500 & 0.1800 & 0.027 & 0.283 & 8.2 & 8.1 & 11.5 & 0.281 & 0.6 & 0.4 & 0.2 & 0.0 \\
\hline 500 & 0.2500 & 0.020 & 0.254 & 10.5 & 9.5 & 14.2 & 0.253 & 0.5 & 0.4 & 0.1 & 0.0 \\
\hline 500 & 0.4000 & 0.012 & 0.139 & 15.4 & 15.1 & 21.6 & 0.138 & 0.5 & 0.4 & 0.1 & 0.0 \\
\hline 500 & 0.6500 & 0.008 & 0.026 & 19.6 & 10.9 & 22.4 & 0.026 & 0.4 & 0.4 & 0.1 & 0.0 \\
\hline 650 & 0.0130 & 0.492 & 0.988 & 6.0 & 4.1 & 7.3 & 0.995 & -0.7 & 0.7 & 0.8 & -2.2 \\
\hline 650 & 0.0200 & 0.320 & 0.791 & 6.3 & 4.4 & 7.7 & 0.785 & 0.7 & 0.7 & 0.7 & -0.7 \\
\hline 650 & 0.0320 & 0.200 & 0.684 & 6.1 & 4.3 & 7.4 & 0.677 & 1.1 & 0.7 & 0.6 & -0.2 \\
\hline 650 & 0.0500 & 0.128 & 0.538 & 6.5 & 5.2 & 8.3 & 0.532 & 1.2 & 0.7 & 0.5 & -0.1 \\
\hline 650 & 0.0800 & 0.080 & 0.436 & 7.1 & 5.8 & 9.2 & 0.431 & 1.1 & 0.7 & 0.4 & 0.0 \\
\hline 650 & 0.1300 & 0.049 & 0.343 & 8.8 & 5.8 & 10.5 & 0.339 & 1.0 & 0.6 & 0.3 & 0.0 \\
\hline 650 & 0.1800 & 0.036 & 0.330 & 9.1 & 7.5 & 11.8 & 0.3 & 0 . & 0.6 & 0.3 & 0.0 \\
\hline 650 & 0.2500 & 0.026 & 0.251 & 11.9 & 10.6 & 15.9 & 0.249 & 0.8 & 0.6 & 0.2 & 0.0 \\
\hline 650 & 0.4000 & 0.016 & 0.090 & 22.9 & 9.6 & 24.9 & 0.090 & 0.7 & 0.5 & 0.1 & 0.0 \\
\hline 800 & 0.0130 & 0.606 & 0.842 & 10.2 & 5.8 & 11.7 & 0.854 & -1.4 & 1.0 & 1.1 & -3.5 \\
\hline 800 & 0.0200 & 0.394 & 0.806 & 7.2 & 4.9 & 8.8 & 0.799 & 0.9 & 1.0 & 1.0 & -1.1 \\
\hline 800 & 0.0320 & 0.246 & 0.721 & 7.1 & 5.0 & 8.7 & 0.709 & 1.6 & 1.0 & 0.9 & -0.3 \\
\hline 800 & 0.0500 & 0.158 & 0.587 & 7.4 & 4.4 & 8.6 & 0.577 & 1.6 & 0.9 & 0.8 & -0.1 \\
\hline 800 & 0.0800 & 0.099 & 0.518 & 7.8 & 5.2 & 9.4 & 0.510 & 1.5 & 0.9 & 0.7 & 0.0 \\
\hline 800 & 0.1300 & 0.061 & 0.411 & 10.0 & 6.2 & 11.8 & 0.406 & 1.3 & 0.9 & 0.5 & 0.0 \\
\hline 800 & 0.1800 & 0.044 & 0.302 & 11.6 & 6.7 & 13.4 & 0.298 & 1.2 & 0.8 & 0.4 & 0.0 \\
\hline 800 & 0.2500 & 0.032 & 0.212 & 14.1 & 8.2 & 16.4 & 0.210 & 1.1 & 0.8 & 0.3 & 0.0 \\
\hline 800 & 0.4000 & 0.020 & 0.117 & 20.9 & 12.6 & 24.4 & 0.116 & 0.9 & 0.7 & 0.2 & 0.0 \\
\hline 800 & 0.6500 & 0.012 & 0.015 & 21.8 & 14.9 & 26.5 & 0.015 & 0.8 & 0.7 & 0.1 & 0.0 \\
\hline 1000 & 0.0130 & 0.757 & 0.773 & 11.5 & 6.9 & 13.5 & 0.795 & -2.8 & 1.4 & 1.7 & -5.8 \\
\hline 1000 & 0.0200 & 0.492 & 0.787 & 7.9 & 4.7 & 9.2 & 0.778 & 1.2 & 1.4 & 1.6 & -1.8 \\
\hline 1000 & 0.0320 & 0.308 & 0.572 & 9.0 & 4.4 & 10.0 & 0.560 & 2.3 & 1.3 & 1.4 & -0.5 \\
\hline 1000 & 0.0500 & 0.197 & 0.577 & 8.4 & 4.5 & 9.5 & 0.564 & 2.4 & 1.3 & 1.2 & -0.2 \\
\hline 1000 & 0.0800 & 0.123 & 0.450 & 9.3 & 5.6 & 10.8 & 0.440 & 2.2 & 1.2 & 1.0 & 0.0 \\
\hline 1000 & 0.1300 & 0.076 & 0.491 & 10.3 & 5.3 & 11.6 & 0.482 & 1.9 & 1.2 & 0.8 & 0.0 \\
\hline
\end{tabular}




\begin{tabular}{|c|c|c|c|c|c|c|c|c|c|c|c|}
\hline $\begin{array}{r}Q^{2} \\
\left(\mathrm{GeV}^{2}\right) \\
\end{array}$ & $x$ & $y$ & $\tilde{\sigma}_{N C}$ & $\begin{array}{l}\delta_{\text {sta }} \\
(\%) \\
\end{array}$ & $\begin{array}{l}\delta_{\text {sys }} \\
(\%) \\
\end{array}$ & $\begin{array}{r}\delta_{t o t} \\
(\%) \\
\end{array}$ & $F_{2}$ & $\begin{array}{l}\Delta_{\text {all }} \\
(\%) \\
\end{array}$ & $\begin{array}{l}\Delta_{F_{2}} \\
(\%) \\
\end{array}$ & $\begin{array}{l}\Delta_{F_{3}} \\
(\%) \\
\end{array}$ & $\begin{array}{r}\Delta_{F_{L}} \\
(\%) \\
\end{array}$ \\
\hline 1000 & 0.1800 & 0.055 & 0.249 & 13.5 & 5.7 & 14.6 & 0.245 & 1.7 & $\bar{~} 1.1$ & 0.6 & 0.0 \\
\hline 1000 & 0.2500 & 0.039 & 0.311 & 13.0 & 9.2 & 15.9 & 0.306 & 1.5 & 1.1 & 0.5 & 0.0 \\
\hline 1000 & 0.4000 & 0.025 & 0.122 & 22.9 & 14.0 & 26.9 & 0.120 & 1.3 & 1.0 & 0.3 & 0.0 \\
\hline 1200 & 0.0200 & 0.591 & 0.839 & 9.1 & 4.0 & 10.0 & 0.829 & 1.3 & 1.8 & 2.3 & -2.7 \\
\hline 1200 & 0.0320 & 0.369 & 0.719 & 9.2 & 3.7 & 9.9 & 0.698 & 3.0 & 1.7 & 2.1 & -0.7 \\
\hline 1200 & 0.0500 & 0.236 & 0.645 & 9.3 & 3.6 & 9.9 & 0.624 & 3.2 & 1.7 & 1.8 & -0.2 \\
\hline 1200 & 0.0800 & 0.148 & 0.415 & 10.7 & 3.4 & 11.2 & 0.403 & 3.0 & 1.6 & 1.4 & -0.1 \\
\hline 1200 & 0.1300 & 0.091 & 0.384 & 12.6 & 4.5 & 13.4 & 0.375 & 2.6 & 1.5 & 1.1 & 0.0 \\
\hline 1200 & 0.1800 & 0.066 & 0.341 & 13.6 & 5.3 & 14.6 & 0.333 & 2.3 & 1.4 & 0.9 & 0.0 \\
\hline 1200 & 0.2500 & 0.047 & 0.251 & 15.8 & 7.0 & 17.3 & 0.246 & 2.0 & 1.4 & 0.7 & 0.0 \\
\hline 1200 & 0.4000 & 0.030 & 0.110 & 25.0 & 12.0 & 27.7 & 0.109 & 1.7 & 1.3 & 0.4 & 0.0 \\
\hline 1500 & 0.0200 & 0.738 & 0.860 & 12.4 & 5.5 & 13.5 & 0.850 & 1.2 & 2.4 & 3.4 & -4.6 \\
\hline 1500 & 0.0320 & 0.462 & 0.704 & 10.4 & 4.7 & 11.4 & 0.675 & 4.3 & 2.4 & 3.2 & -1.2 \\
\hline 1500 & 0.0500 & 0.295 & 0.515 & 11.7 & 3.6 & 12.2 & .492 & 4.7 & 2.3 & 2.7 & -0.4 \\
\hline 1500 & 0.0800 & 0.185 & 0.512 & 11.0 & 4.0 & 11.7 & 0.490 & 4.3 & 2.2 & 2.2 & -0.1 \\
\hline 1500 & 0.1300 & 0.114 & 0.390 & 13.9 & 5.0 & 14.8 & 0.376 & 3.7 & 2.1 & 1.7 & 0.0 \\
\hline 1500 & 0.1800 & 0.082 & 0.260 & 18.6 & 4.3 & 19.1 & 0.251 & 3.3 & 2.0 & 1.3 & 0.0 \\
\hline 1500 & 0.2500 & 0.059 & 0.197 & 19.6 & 7.7 & 21.1 & 0.191 & 2.9 & 1.9 & 1.0 & 0.0 \\
\hline 1500 & 0.4000 & 0.037 & 0.145 & 24.3 & 12.8 & 27.4 & 0.142 & 2.4 & 1.7 & 0.7 & 0.0 \\
\hline 1500 & 0.6500 & 0.023 & 0.014 & 35.4 & 16.1 & 38.9 & 0.013 & 2.0 & 1.6 & 0.4 & 0.0 \\
\hline 2000 & 0.0320 & 0.615 & 0.796 & 11.1 & 4.4 & 11.9 & 0.747 & 6.6 & 3.6 & 5.4 & -2.4 \\
\hline 2000 & 0.0500 & 0.394 & 0.599 & 13.0 & 5.0 & 13.9 & 0.557 & 7.6 & 3.5 & 4.8 & -0.7 \\
\hline 2000 & 0.0800 & 0.246 & 0.582 & 12.3 & 4.3 & 13.0 & 0.544 & 7.0 & 3.3 & 3.9 & -0.2 \\
\hline 2000 & 0.1300 & 0.152 & 0.224 & 20.0 & 4.6 & 20.6 & 0.212 & 6.0 & 3.1 & 2.9 & 0.0 \\
\hline 2000 & 0.1800 & 0.109 & 0.249 & 21.9 & 6.3 & 22.7 & 0.236 & 5.2 & 3.0 & 2.3 & 0.0 \\
\hline 2000 & 0.2500 & 0.079 & 0.197 & 22.4 & 6.8 & 23.4 & 0.188 & 4.5 & 2.8 & 1.8 & 0.0 \\
\hline 2000 & 0.4000 & 0.049 & 0.108 & 27.7 & 10.1 & 29.5 & 0.104 & 3.7 & 2.6 & 1.1 & 0.0 \\
\hline 3000 & 0.0500 & 0.591 & 0.606 & 10.6 & 6.4 & 12.4 & 0.530 & 14.4 & 6.0 & 10.0 & -1.7 \\
\hline 3000 & 0.0800 & 0.369 & 0.556 & 10.9 & 4.5 & 11.8 & 0.489 & 13.6 & 5.7 & 8.3 & -0.4 \\
\hline 3000 & 0.1300 & 0.227 & 0.464 & 12.4 & 4.0 & 13.0 & 0.416 & 11.4 & 5.4 & 6.2 & -0.1 \\
\hline 3000 & 0.1800 & 0.164 & 0.347 & 15.3 & 5.1 & 16.1 & 0.315 & 9.9 & 5.1 & 4.8 & 0.0 \\
\hline 3000 & 0.2500 & 0.118 & 0.255 & 17.8 & 7.0 & 19.1 & 0.235 & 8.5 & 4.8 & 3.7 & 0.0 \\
\hline 3000 & 0.4000 & 0.074 & 0.128 & 23.0 & 10.9 & 25.5 & 0.120 & 6.8 & 4.4 & 2.4 & 0.0 \\
\hline 5000 & 0.0800 & 0.615 & 0.707 & 10.6 & 4.8 & 11.7 & 0.545 & 29.7 & 10.8 & 20.3 & -1.4 \\
\hline 5000 & 0.1300 & 0.379 & 0.536 & 13.1 & 5.3 & 14.2 & 0.428 & 25.2 & 10.1 & 15.4 & -0.3 \\
\hline 5000 & 0.1800 & 0.274 & 0.442 & 14.0 & 5.2 & 14.9 & 0.364 & 21.5 & 9.6 & 12.0 & -0.1 \\
\hline 5000 & 0.2500 & 0.197 & 0.361 & 17.4 & 10.5 & 20.3 & 0.306 & 18.0 & 9.0 & 9.0 & 0.0 \\
\hline 5000 & 0.4000 & 0.123 & 0.091 & 31.6 & 11.1 & 33.5 & 0.080 & 14.0 & 8.3 & 5.7 & 0.0 \\
\hline
\end{tabular}




\begin{tabular}{|r|c|c||c|r|r|r||r|r|r|r|r|}
\hline $\begin{array}{r}Q^{2} \\
\left(\mathrm{GeV}^{2}\right)\end{array}$ & $x$ & $y$ & $\tilde{\sigma}_{N C}$ & $\begin{array}{r}\delta_{\text {sta }} \\
(\%)\end{array}$ & $\begin{array}{r}\delta_{\text {sys }} \\
(\%)\end{array}$ & $\begin{array}{r}\delta_{\text {tot }} \\
(\%)\end{array}$ & $F_{2}$ & $\begin{array}{r}\Delta_{\text {all }} \\
(\%)\end{array}$ & $\begin{array}{r}\Delta_{F_{2}} \\
(\%)\end{array}$ & $\begin{array}{r}\Delta_{F_{3}} \\
(\%)\end{array}$ & $\begin{array}{r}\Delta_{F_{L}} \\
(\%)\end{array}$ \\
\hline \hline 5000 & 0.6500 & 0.076 & 0.010 & 41.0 & 18.8 & 45.1 & 0.009 & 11.1 & 7.6 & 3.5 & 0.0 \\
\hline 8000 & 0.1300 & 0.606 & 0.722 & 16.0 & 6.5 & 17.2 & 0.485 & 49.0 & 16.6 & 33.3 & -0.9 \\
8000 & 0.1800 & 0.438 & 0.386 & 20.4 & 5.8 & 21.2 & 0.272 & 41.8 & 15.7 & 26.4 & -0.3 \\
8000 & 0.2500 & 0.315 & 0.295 & 21.8 & 8.2 & 23.3 & 0.219 & 34.5 & 14.8 & 19.8 & -0.1 \\
8000 & 0.4000 & 0.197 & 0.197 & 27.7 & 16.8 & 32.4 & 0.156 & 26.0 & 13.6 & 12.5 & 0.0 \\
\hline 12000 & 0.1800 & 0.656 & 0.471 & 27.8 & 7.6 & 28.8 & 0.277 & 70.0 & 22.3 & 48.6 & -0.9 \\
12000 & 0.2500 & 0.473 & 0.298 & 28.9 & 8.6 & 30.2 & 0.189 & 58.1 & 21.0 & 37.4 & -0.3 \\
12000 & 0.4000 & 0.295 & 0.083 & 50.0 & 19.6 & 53.7 & 0.058 & 42.7 & 19.3 & 23.5 & -0.1 \\
\hline 20000 & 0.2500 & 0.788 & 0.349 & 51.1 & 10.8 & 52.2 & 0.174 & 101.1 & 29.4 & 72.8 & -1.1 \\
20000 & 0.4000 & 0.492 & 0.182 & 44.7 & 13.3 & 46.7 & 0.103 & 76.3 & 27.0 & 49.5 & -0.2 \\
20000 & 0.6500 & 0.303 & 0.014 & 70.7 & 36.9 & 79.8 & 0.009 & 54.2 & 24.8 & 29.4 & 0.0 \\
\hline 30000 & 0.4000 & 0.738 & 0.268 & 70.7 & 17.5 & 72.9 & 0.125 & 113.7 & 32.8 & 81.4 & -0.6 \\
\hline
\end{tabular}




\begin{tabular}{|c|c|c|c|c|c|c|c|r|}
\hline $\begin{array}{c}Q^{2} \\
\left(\mathrm{GeV}^{2}\right)\end{array}$ & $x$ & $y$ & $\begin{array}{c}\mathrm{d}^{2} \sigma_{C C} / \mathrm{d} x \mathrm{~d} Q^{2} \\
\left(\mathrm{pb} / \mathrm{GeV}^{2}\right)\end{array}$ & $\phi_{C C}$ & $\begin{array}{c}\delta_{\text {sta }} \\
(\%)\end{array}$ & $\begin{array}{c}\delta_{\text {sys }} \\
(\%)\end{array}$ & $\begin{array}{c}\delta_{\text {tot }} \\
(\%)\end{array}$ & $\begin{array}{r}\delta_{C C}^{\text {qed }} \\
(\%)\end{array}$ \\
\hline 300 & 0.013 & 0.227 & $0.458 \cdot 10^{0}$ & 0.773 & 55.4 & 15.7 & 57.6 & 3.5 \\
300 & 0.032 & 0.092 & $0.399 \cdot 10^{0}$ & 1.658 & 24.5 & 12.0 & 27.3 & 5.6 \\
300 & 0.080 & 0.037 & $0.690 \cdot 10^{-1}$ & 0.717 & 40.7 & 11.6 & 42.3 & 7.0 \\
\hline 500 & 0.013 & 0.379 & $0.433 \cdot 10^{0}$ & 0.775 & 37.6 & 13.3 & 39.9 & -0.3 \\
500 & 0.032 & 0.154 & $0.285 \cdot 10^{0}$ & 1.257 & 19.6 & 7.8 & 21.0 & 5.0 \\
500 & 0.080 & 0.061 & $0.790 \cdot 10^{-1}$ & 0.870 & 21.8 & 5.1 & 22.4 & 1.4 \\
500 & 0.130 & 0.038 & $0.551 \cdot 10^{-1}$ & 0.986 & 29.0 & 7.0 & 29.9 & 3.3 \\
\hline 1000 & 0.032 & 0.308 & $0.186 \cdot 10^{0}$ & 0.941 & 17.5 & 4.9 & 18.2 & -3.8 \\
1000 & 0.080 & 0.123 & $0.556 \cdot 10^{-1}$ & 0.703 & 17.9 & 4.3 & 18.4 & 2.1 \\
1000 & 0.130 & 0.076 & $0.310 \cdot 10^{-1}$ & 0.637 & 24.0 & 4.6 & 24.5 & 3.4 \\
1000 & 0.250 & 0.039 & $0.139 \cdot 10^{-1}$ & 0.548 & 37.6 & 10.6 & 39.1 & -1.7 \\
\hline 2000 & 0.032 & 0.615 & $0.132 \cdot 10^{0}$ & 0.859 & 15.5 & 4.9 & 16.2 & -2.0 \\
2000 & 0.080 & 0.246 & $0.571 \cdot 10^{-1}$ & 0.929 & 13.0 & 3.9 & 13.6 & -2.5 \\
2000 & 0.130 & 0.152 & $0.197 \cdot 10^{-1}$ & 0.521 & 21.2 & 4.5 & 21.7 & -0.2 \\
2000 & 0.250 & 0.079 & $0.855 \cdot 10^{-2}$ & 0.435 & 25.6 & 6.5 & 26.4 & -0.1 \\
\hline 3000 & 0.080 & 0.369 & $0.324 \cdot 10^{-1}$ & 0.659 & 14.0 & 4.8 & 14.8 & 0.1 \\
3000 & 0.130 & 0.227 & $0.250 \cdot 10^{-1}$ & 0.827 & 14.0 & 6.1 & 15.2 & -0.7 \\
3000 & 0.250 & 0.118 & $0.749 \cdot 10^{-2}$ & 0.476 & 18.9 & 7.0 & 20.1 & -1.5 \\
3000 & 0.400 & 0.074 & $0.251 \cdot 10^{-2}$ & 0.255 & 35.2 & 19.6 & 40.3 & -1.1 \\
\hline 5000 & 0.080 & 0.615 & $0.213 \cdot 10^{-1}$ & 0.637 & 17.9 & 6.7 & 19.2 & -5.3 \\
5000 & 0.130 & 0.379 & $0.108 \cdot 10^{-1}$ & 0.525 & 16.8 & 7.0 & 18.2 & -3.8 \\
5000 & 0.250 & 0.197 & $0.550 \cdot 10^{-2}$ & 0.512 & 16.3 & 4.4 & 16.9 & -2.9 \\
5000 & 0.400 & 0.123 & $0.123 \cdot 10^{-2}$ & 0.183 & 33.1 & 13.1 & 35.6 & -4.9 \\
\hline 8000 & 0.130 & 0.606 & $0.722 \cdot 10^{-2}$ & 0.557 & 18.9 & 9.3 & 21.1 & -9.9 \\
8000 & 0.250 & 0.315 & $0.342 \cdot 10^{-2}$ & 0.508 & 16.3 & 6.2 & 17.4 & -5.0 \\
8000 & 0.400 & 0.197 & $0.946 \cdot 10^{-3}$ & 0.225 & 28.6 & 10.3 & 30.4 & -7.9 \\
\hline 15000 & 0.250 & 0.591 & $0.139 \cdot 10^{-2}$ & 0.453 & 22.1 & 16.0 & 27.3 & -10.9 \\
15000 & 0.400 & 0.369 & $0.419 \cdot 10^{-3}$ & 0.219 & 27.5 & 10.7 & 29.5 & -17.7 \\
\hline
\end{tabular}

Table 7: The CC double differential cross section $\mathrm{d}^{2} \sigma_{C C} / \mathrm{d} x \mathrm{~d} Q^{2}$ and the structure function term $\phi_{C C}\left(x, Q^{2}\right)$ computed assuming $M_{W}=80.41 \mathrm{GeV}$. Also given are the statistical $\left(\delta_{\text {sta }}\right)$, systematic $\left(\delta_{\text {sys }}\right)$, and total $\left(\delta_{\text {tot }}\right)$ errors. The last column gives the correction for $Q E D$ radiative effects $\delta_{C C}^{q e d}$. The normalisation uncertainty of $1.8 \%$ is not included in the errors. 


\begin{tabular}{|c|c|c|c|c|c|c|c|c|c|c|c|c|c|}
\hline $\begin{array}{r}Q^{2} \\
\left(\mathrm{GeV}^{2}\right) \\
\end{array}$ & $x$ & $\tilde{\sigma}_{N C}$ & $\begin{array}{r}\delta_{t o t} \\
(\%)\end{array}$ & $\begin{array}{r}\delta_{\text {sta }} \\
(\%) \\
\end{array}$ & $\begin{array}{r}\delta_{u n c} \\
(\%) \\
\end{array}$ & $\begin{array}{c}\delta_{u n c}^{E} \\
(\%) \\
\end{array}$ & $\begin{array}{r}\delta_{u n c}^{h} \\
(\%) \\
\end{array}$ & $\begin{array}{r}\delta_{c o r} \\
(\%) \\
\end{array}$ & $\begin{array}{c}\delta_{c o r}^{E^{+}} \\
(\%) \\
\end{array}$ & $\begin{array}{c}\delta_{c o r}^{\theta^{+}} \\
(\%) \\
\end{array}$ & $\begin{array}{c}\delta_{c o r}^{h^{+}} \\
(\%)\end{array}$ & $\begin{array}{c}\delta_{\text {cor }}^{N^{+}} \\
(\%)\end{array}$ & $\begin{array}{c}\delta_{\text {cor }}^{B^{+}} \\
(\%) \\
\end{array}$ \\
\hline$\overline{\overline{150}}$ & $\overline{0.0032}$ & 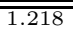 & $\overline{4.7}$ & $\overline{2.7}$ & 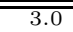 & $\overline{0.5}$ & $\overline{\overline{0.6}}$ & $\overline{2.3}$ & $\overline{\overline{0.1}}$ & $=1.8$ & $\overline{\overline{1.3}}$ & $\overline{\overline{0.1}}$ & -0.7 \\
\hline 150 & 0.0050 & 1.154 & 4.4 & 2.8 & 3.0 & 0.2 & 0.5 & 1.5 & 0.4 & 1.4 & 0.3 & 0.2 & -0.1 \\
\hline 150 & 0.0080 & 0.968 & 9.1 & 4.1 & 5.4 & 4.0 & 1.0 & 6.1 & -1.6 & 5.9 & -0.7 & 0.1 & 0.0 \\
\hline 200 & 0.0032 & 1.271 & 6.1 & 4.1 & 3.5 & 0.8 & 0.2 & 2.9 & -1.0 & -2.5 & 0.5 & 0.2 & -0.9 \\
\hline 200 & 0.0050 & 1.107 & 4.6 & 2.8 & 3.1 & 0.7 & 0.9 & 1.8 & -0.5 & -1.0 & 1.4 & 0.2 & -0.3 \\
\hline 200 & 0.0080 & 0.915 & 4.5 & 3.0 & 3.2 & 1.1 & 0.4 & 0.9 & 0.4 & -0.6 & 0.4 & -0.2 & -0.1 \\
\hline 200 & 0.0130 & 0.860 & 4.7 & 3.2 & 3.3 & 1.2 & 0.1 & 1.1 & -0.3 & 0.8 & -0.6 & 0.4 & 0.0 \\
\hline 200 & 0.0200 & 0.677 & 6.5 & 3.8 & 4.5 & 3.1 & 0.1 & 2.9 & -1.2 & 2.6 & -0.4 & 0.1 & 0.0 \\
\hline 200 & 0.0320 & 0.558 & 8.6 & 4.5 & 6.1 & 5.0 & 0.5 & 4.2 & -2.0 & 3.5 & -1.1 & 0.3 & 0.0 \\
\hline 200 & 0.0500 & 0.506 & 9.9 & 5.2 & 6.8 & 5.7 & 0.7 & 4.9 & -1.6 & 4.5 & -0.6 & -1.1 & 0.0 \\
\hline 200 & 0.0800 & 0.407 & 12.4 & 5.9 & 8.4 & 7.4 & 0.8 & 6.9 & -3.0 & 6.2 & 0.4 & 1.0 & 0.0 \\
\hline 250 & 0.0050 & 1.123 & 5.3 & 3.5 & 3.3 & 1.1 & 0.7 & 2.3 & 0.6 & -1.7 & 1.3 & 0.2 & -0.5 \\
\hline 250 & 0.0080 & 1.021 & 5.3 & 3.2 & 3.7 & 2.0 & 0.7 & 2.0 & -0.8 & -1.6 & 0.8 & 0.1 & 0.0 \\
\hline 250 & 0.0130 & 0.825 & 5.7 & 3.4 & 4.0 & 2.6 & 0.5 & 2.1 & 0.6 & -2.0 & 0.3 & 0.3 & 0.0 \\
\hline 250 & 0.0200 & 0.691 & 5.4 & 3.5 & 3.6 & 1.8 & 0.5 & 1.8 & 0.6 & -1.7 & -0.2 & 0.5 & 0.0 \\
\hline 250 & 0.0320 & 0.569 & 6.1 & 3.8 & 3.9 & 2.3 & 0.7 & 2.6 & 1.0 & -2.2 & -0.9 & -0.3 & 0.0 \\
\hline 250 & 0.0500 & 0.493 & 5.7 & 4.3 & 3.4 & 0.8 & 0.5 & 1.4 & -0.2 & -1.1 & -0.6 & 0.6 & 0.0 \\
\hline 250 & 0.0800 & 0.407 & 6.1 & 4.7 & 3.5 & 1.0 & 0.6 & 1.6 & -0.6 & -0.4 & 0.3 & 1.4 & 0.0 \\
\hline 250 & 0.1300 & 0.311 & 7.8 & 5.3 & 5.2 & 3.1 & 2.2 & 2.6 & 1.6 & -0.9 & -1.4 & -1.3 & 0.0 \\
\hline 250 & 0.2500 & 0.225 & 12.1 & 7.5 & 8.6 & 6.9 & 2.8 & 4.1 & 2.4 & -1.5 & -1.8 & -2.3 & 0.0 \\
\hline 250 & 0.4000 & 0.138 & 11.8 & 9.3 & 6.8 & 4.2 & 1.4 & 2.3 & 0.4 & -1.0 & -0.4 & -2.0 & 0.0 \\
\hline 300 & 0.0050 & 1.152 & 7.2 & 5.6 & 3.9 & 1.2 & 0.3 & 2.3 & 0.4 & -2.2 & 0.2 & 0.1 & -0.6 \\
\hline 300 & 0.0080 & 1.026 & 5.1 & 3.6 & 3.3 & 0.2 & 1.0 & 1.5 & -0.6 & -0.8 & 1.1 & 0.1 & -0.1 \\
\hline 300 & 0.0130 & 0.878 & 5.3 & 3.8 & 3.3 & 0.7 & 0.8 & 1.7 & -0.3 & -1.5 & 0.7 & 0.2 & 0.0 \\
\hline 300 & 0.0200 & 0.735 & 5.9 & 4.0 & 3.9 & 2.1 & 0.3 & 1.8 & 0.9 & -1.5 & -0.1 & 0.3 & -0.1 \\
\hline 300 & 0.0320 & 0.605 & 5.8 & 4.2 & 3.8 & 2.0 & 0.3 & 1.5 & 0.7 & -1.3 & -0.2 & 0.3 & 0.0 \\
\hline 300 & 0.0500 & 0.509 & 6.8 & 4.5 & 4.5 & 3.1 & 0.1 & 2.3 & 1.0 & -2.0 & 0.2 & 0.6 & 0.0 \\
\hline 300 & 0.0800 & 0.390 & 6.9 & 5.2 & 4.1 & 2.3 & 0.3 & 2.0 & 0.9 & -1.7 & -0.3 & 0.5 & 0.0 \\
\hline 300 & 0.1300 & 0.332 & 8.8 & 5.4 & 6.7 & 5.6 & 1.1 & 2.1 & 1.1 & -1.6 & -0.7 & -0.4 & 0.0 \\
\hline 300 & 0.2500 & 0.277 & 12.8 & 6.9 & 9.4 & 7.7 & 3.4 & 5.3 & 3.1 & -2.6 & -2.1 & -2.7 & 0.0 \\
\hline 300 & 0.4000 & 0.143 & 14.2 & 10.3 & 8.9 & 6.8 & 1.9 & 4.2 & 2.8 & -2.6 & -0.8 & -1.6 & 0.0 \\
\hline 400 & 0.0080 & 1.088 & 6.1 & 4.5 & 3.5 & 0.2 & 0.9 & 2.2 & 0.3 & -1.8 & 1.3 & 0.1 & -0.2 \\
\hline 400 & 0.0130 & 0.897 & 5.6 & 4.3 & 3.4 & 0.3 & 0.8 & 1.2 & 0.7 & -0.8 & 0.6 & 0.2 & 0.0 \\
\hline 400 & 0.0200 & 0.732 & 5.8 & 4.5 & 3.5 & 1.0 & 0.4 & 1.1 & 0.2 & -0.9 & -0.5 & 0.2 & 0.0 \\
\hline 400 & 0.0320 & 0.560 & 6.1 & 4.8 & 3.6 & 1.2 & 0.4 & 1.3 & 0.6 & -1.1 & 0.5 & 0.1 & -0.1 \\
\hline 400 & 0.0500 & 0.514 & 6.3 & 5.0 & 3.6 & 1.0 & 0.3 & 1.0 & 0.4 & 0.6 & -0.6 & 0.4 & 0.0 \\
\hline 400 & 0.0800 & 0.429 & 7.0 & 5.5 & 3.8 & 1.4 & 0.5 & 2.1 & 1.5 & -1.2 & -0.1 & 0.8 & 0.0 \\
\hline 400 & 0.1300 & 0.352 & 7.5 & 5.6 & 4.4 & 2.4 & 1.1 & 2.2 & 1.5 & -1.4 & -0.7 & -0.1 & 0.0 \\
\hline 400 & 0.2500 & 0.240 & 10.6 & 7.6 & 6.7 & 4.5 & 2.7 & 3.2 & 2.1 & -0.8 & -1.6 & -1.6 & 0.0 \\
\hline 400 & 0.4000 & 0.143 & 13.7 & 10.8 & 7.3 & 4.1 & 2.4 & 4.1 & 1.9 & -1.8 & -1.3 & -2.8 & 0.0 \\
\hline 500 & 0.0080 & 1.044 & 9.3 & 7.8 & 4.6 & 1.3 & 0.5 & 2.0 & -0.8 & -1.6 & 0.9 & -0.2 & -0.5 \\
\hline 500 & 0.0130 & 1.003 & 6.8 & 5.1 & 3.9 & 1.2 & 1.1 & 2.3 & 0.6 & -2.1 & 1.0 & 0.2 & -0.1 \\
\hline 500 & 0.0200 & 0.765 & 7.0 & 5.1 & 4.2 & 2.1 & 1.2 & 2.3 & 0.9 & -1.9 & 0.8 & 0.2 & 0.0 \\
\hline 500 & 0.0320 & 0.604 & 7.0 & 5.3 & 3.8 & 1.5 & 0.5 & 2.5 & 1.3 & -2.0 & 0.2 & 0.5 & 0.0 \\
\hline 500 & 0.0500 & 0.517 & 6.9 & 5.6 & 3.8 & 1.3 & 0.6 & 1.4 & 0.5 & -1.2 & -0.2 & 0.5 & 0.0 \\
\hline 500 & 0.0800 & 0.392 & 9.2 & 6.4 & 5.8 & 4.3 & 1.3 & 3.0 & 2.0 & -2.0 & -0.8 & -0.3 & 0.0 \\
\hline 500 & 0.1300 & 0.363 & 8.7 & 7.2 & 4.1 & 0.4 & 0.8 & 2.7 & 0.5 & -1.7 & -0.5 & 2.0 & 0.0 \\
\hline 500 & 0.1800 & 0.283 & 11.5 & 8.2 & 6.6 & 4.5 & 2.4 & 4.7 & 3.5 & -2.1 & -1.6 & -1.5 & 0.0 \\
\hline 500 & 0.2500 & 0.254 & 14.2 & 10.5 & 8.4 & 6.0 & 2.9 & 4.5 & 3.3 & -2.4 & -1.6 & -1.1 & 0.0 \\
\hline 500 & 0.4000 & 0.139 & 21.6 & 15.4 & 12.0 & 8.5 & 3.9 & 9.2 & 7.2 & -3.5 & -2.3 & -3.9 & 0.0 \\
\hline 500 & 0.6500 & 0.026 & 22.4 & 19.6 & 10.5 & 2.8 & 2.4 & 3.0 & -1.5 & -0.8 & -2.1 & -1.3 & 0.0 \\
\hline 650 & 0.0130 & 0.988 & 7.3 & 6.0 & 4.0 & 0.5 & 0.9 & 1.1 & -0.4 & -0.3 & 0.9 & 0.1 & -0.2 \\
\hline 650 & 0.0200 & 0.791 & 7.7 & 6.3 & 4.2 & 1.2 & 0.9 & 1.5 & 0.6 & -0.9 & 1.0 & -0.3 & 0.0 \\
\hline 650 & 0.0320 & 0.684 & 7.4 & 6.1 & 4.0 & 0.7 & 1.0 & 1.6 & 0.5 & -1.4 & 0.6 & 0.3 & 0.0 \\
\hline 650 & 0.0500 & 0.538 & 8.3 & 6.5 & 4.5 & 2.2 & 0.3 & 2.7 & 1.9 & -1.8 & 0.2 & 0.5 & 0.0 \\
\hline 650 & 0.0800 & 0.436 & 9.2 & 7.1 & 5.0 & 2.7 & 1.2 & 3.1 & 2.2 & -1.6 & -1.2 & -0.3 & 0.0 \\
\hline 650 & 0.1300 & 0.343 & 10.5 & 8.8 & 5.4 & 2.1 & 2.2 & 2.3 & 1.5 & -0.6 & 1.0 & 1.2 & 0.0 \\
\hline 650 & 0.1800 & 0.330 & 11.8 & 9.1 & 6.8 & 4.4 & 2.0 & 3.2 & 2.1 & -1.6 & -1.7 & -0.5 & 0.0 \\
\hline 650 & 0.2500 & 0.251 & 15.9 & 11.9 & 9.3 & 6.3 & 3.9 & 5.0 & 3.6 & -1.1 & -2.1 & -2.7 & 0.0 \\
\hline 650 & 0.4000 & 0.090 & 24.9 & 22.9 & 9.3 & 3.2 & 1.6 & 2.4 & 1.9 & 0.9 & 1.2 & 0.2 & 0.0 \\
\hline 800 & 0.0130 & 0.842 & 11.7 & 10.2 & 5.2 & 0.6 & 0.2 & 2.5 & 1.3 & -2.2 & -0.3 & 0.0 & -0.2 \\
\hline 800 & 0.0200 & 0.806 & 8.8 & 7.2 & 4.6 & 1.3 & 0.9 & 1.7 & 1.1 & 1.1 & 0.5 & 0.0 & -0.5 \\
\hline 800 & 0.0320 & 0.721 & 8.7 & 7.1 & 4.8 & 1.9 & 1.2 & 1.3 & 0.8 & -0.2 & 1.1 & 0.2 & 0.0 \\
\hline 800 & 0.0500 & 0.587 & 8.6 & 7.4 & 4.4 & 0.9 & 0.7 & 0.8 & -0.3 & 0.1 & -0.6 & 0.3 & 0.0 \\
\hline 800 & 0.0800 & 0.518 & 9.4 & 7.8 & 4.9 & 1.9 & 0.7 & 1.6 & 1.1 & 0.6 & -1.0 & 0.4 & 0.0 \\
\hline 800 & 0.1300 & 0.411 & 11.8 & 10.0 & 5.8 & 2.5 & 0.6 & 2.2 & 1.5 & -1.4 & -0.5 & 0.5 & 0.0 \\
\hline 800 & 0.1800 & 0.302 & 13.4 & 11.6 & 6.2 & 2.5 & 1.6 & 2.7 & 1.9 & -1.4 & -0.9 & 0.8 & 0.0 \\
\hline 800 & 0.2500 & 0.212 & 16.4 & 14.1 & 7.7 & 4.3 & 2.0 & 2.9 & 2.0 & -0.9 & -1.0 & -1.5 & 0.0 \\
\hline 800 & 0.4000 & 0.117 & 24.4 & 20.9 & 11.6 & 6.6 & 3.3 & 5.1 & 3.7 & -2.2 & -2.1 & -1.6 & 0.0 \\
\hline 800 & 0.6500 & 0.015 & 26.5 & 21.8 & 12.5 & 9.0 & 3.9 & 8.1 & 6.3 & -3.9 & -1.6 & -3.1 & 0.0 \\
\hline
\end{tabular}

Table 8: The NC reduced cross section $\tilde{\sigma}_{N C}\left(x, Q^{2}\right)$ with statistical $\left(\delta_{\text {sta }}\right)$, total $\left(\delta_{t o t}\right)$, and uncorrelated systematic $\left(\delta_{\text {unc }}\right)$ errors, and its contributions from the electron energy error $\left(\delta_{\text {unc }}^{E}\right)$, and the hadronic energy error $\left(\delta_{u n c}^{h}\right)$. The effect of the other uncorrelated systematic errors is included in $\left(\delta_{u n c}\right)$. In addition the correlated systematic $\left(\delta_{c o r}\right)$, and its contributions from a positive variation of one standard deviation of the electron energy error $\left(\delta_{c o r}^{E^{+}}\right)$, of the polar electron angle error $\left(\delta_{c o r}^{\theta^{+}}\right)$, of the hadronic energy error $\left(\delta_{c o r}^{h^{+}}\right)$, of the error due to noise subtraction $\left(\delta_{c o r}^{N^{+}}\right)$, and of the error due to background subtraction $\left(\delta_{c o r}^{B^{+}}\right)$. The normalisation uncertainty of $1.8 \%$ is not included in the errors. The table continues on the next page. 


\begin{tabular}{|c|c|c|c|c|c|c|c|c|c|c|c|c|c|}
\hline $\begin{array}{r}Q^{2} \\
\left(\mathrm{GeV}^{2}\right) \\
\end{array}$ & $x$ & $\tilde{\sigma}_{N C}$ & $\begin{array}{r}\delta_{t o t} \\
(\%)\end{array}$ & $\begin{array}{r}\delta_{\text {sta }} \\
(\%)\end{array}$ & $\begin{array}{r}\delta_{u n c} \\
(\%)\end{array}$ & $\begin{array}{r}\delta_{u n c}^{E} \\
(\%)\end{array}$ & $\begin{array}{r}\delta_{u n c}^{h} \\
(\%) \\
\end{array}$ & $\begin{array}{r}\delta_{\text {cor }} \\
(\%)\end{array}$ & $\begin{array}{c}\delta_{\text {cor }}^{E^{+}} \\
(\%) \\
\end{array}$ & $\begin{array}{c}\delta_{\text {cor }}^{\theta^{+}} \\
(\%)\end{array}$ & $\begin{array}{c}\delta_{\text {cor }}^{h^{+}} \\
(\%) \\
\end{array}$ & $\begin{array}{c}\delta_{\text {cor }}^{N^{+}} \\
(\%)\end{array}$ & $\begin{array}{c}\delta_{\text {cor }}^{B^{+}} \\
(\%) \\
\end{array}$ \\
\hline$\overline{1000}$ & 0.0130 & 0.773 & $\bar{~} 13.5$ & 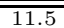 & $\overline{6.5}$ & $\overline{\overline{2.6}}$ & $\overline{1.8}$ & 2.3 & $\overline{c-0.7}$ & $\overline{c-0.8}$ & $\overline{c-1.5}$ & $=-0.2$ & $\overline{c-1.5}$ \\
\hline 1000 & 0.0200 & 0.787 & 9.2 & 7.9 & 4.3 & 1.5 & 1.3 & 1.9 & 1.0 & -0.9 & 1.3 & 0.1 & -0.2 \\
\hline 1000 & 0.0320 & 0.572 & 10.0 & 9.0 & 4.2 & 0.8 & 1.2 & 1.3 & 0.4 & -0.8 & 0.9 & 0.2 & -0.1 \\
\hline 1000 & 0.0500 & 0.577 & 9.5 & 8.4 & 4.3 & 0.5 & 1.4 & 1.3 & 0.1 & -1.0 & 0.5 & 0.5 & 0.0 \\
\hline 1000 & 0.0800 & 0.450 & 10.8 & 9.3 & 4.9 & 2.4 & 1.2 & 2.8 & 2.3 & -1.1 & -1.0 & -0.3 & 0.0 \\
\hline 1000 & 0.1300 & 0.491 & 11.6 & 10.3 & 5.0 & 1.1 & 0.2 & 1.9 & -1.3 & -1.4 & 0.4 & 0.3 & 0.0 \\
\hline 1000 & 0.1800 & 0.249 & 14.6 & 13.5 & 5.4 & 1.9 & 1.1 & 1.6 & 1.2 & -0.8 & -0.3 & -0.4 & 0.0 \\
\hline 1000 & 0.2500 & 0.311 & 15.9 & 13.0 & 8.2 & 5.3 & 2.8 & 4.2 & 2.7 & -2.2 & -2.0 & 1.0 & 0.0 \\
\hline 1000 & 0.4000 & 0.122 & 26.9 & 22.9 & 13.3 & 9.6 & 4.2 & 4.4 & 2.9 & 0.8 & -1.8 & -2.7 & 0.0 \\
\hline 1200 & 0.0200 & 0.839 & 10.0 & 9.1 & 3.6 & 0.7 & 0.8 & 1.8 & -0.6 & -0.9 & 1.3 & 0.0 & -0.6 \\
\hline 1200 & 0.0320 & 0.719 & 9.9 & 9.2 & 3.5 & 0.4 & 1.4 & 1.1 & 0.3 & -0.6 & 0.9 & 0.1 & 0.0 \\
\hline 1200 & 0.0500 & 0.645 & 9.9 & 9.3 & 3.5 & 1.1 & 0.7 & 0.7 & -0.1 & -0.3 & 0.6 & 0.2 & 0.0 \\
\hline 1200 & 0.0800 & 0.415 & 11.2 & 10.7 & 3.4 & 0.8 & 0.6 & 0.6 & 0.4 & -0.2 & 0.2 & 0.3 & 0.0 \\
\hline 1200 & 0.1300 & 0.384 & 13.4 & 12.6 & 4.3 & 2.2 & 0.6 & 1.2 & 1.0 & 0.3 & -0.3 & -0.5 & 0.0 \\
\hline 1200 & 0.1800 & 0.341 & 14.6 & 13.6 & 4.7 & 2.5 & 0.6 & 2.3 & 1.9 & -0.7 & -0.9 & 0.6 & 0.0 \\
\hline 1200 & 0.2500 & 0.251 & 17.3 & 15.8 & 6.4 & 4.5 & 1.8 & 2.9 & 2.6 & -1.1 & -0.5 & 0.2 & 0.0 \\
\hline 1200 & 0.4000 & 0.110 & 27.7 & 25.0 & 10.2 & 7.7 & 4.0 & 6.2 & 4.2 & -1.1 & -3.2 & -3.2 & 0.0 \\
\hline 1500 & 0.0200 & 0.860 & 13.5 & 12.4 & 5.2 & 0.1 & 1.3 & 1.4 & -0.8 & -0.4 & -0.4 & -0.2 & -1.0 \\
\hline 1500 & 0.0320 & 0.704 & 11.4 & 10.4 & 4.3 & 1.5 & 2.2 & 1.8 & -0.6 & -0.6 & 1.6 & 0.2 & -0.1 \\
\hline 1500 & 0.0500 & 0.515 & 12.2 & 11.7 & 3.5 & 0.5 & 0.5 & 0.8 & 0.4 & -0.6 & 0.4 & 0.1 & 0.0 \\
\hline 1500 & 0.0800 & 0.512 & 11.7 & 11.0 & 3.8 & 1.4 & 0.9 & 1.4 & 0.7 & -1.1 & 0.4 & 0.2 & 0.0 \\
\hline 1500 & 0.1300 & 0.390 & 14.8 & 13.9 & 4.7 & 2.8 & 0.7 & 1.8 & 1.6 & -0.7 & 0.4 & -0.2 & 0.0 \\
\hline 1500 & 0.1800 & 0.260 & 19.1 & 18.6 & 4.2 & 0.3 & 1.0 & 1.1 & -0.9 & -0.4 & 0.3 & 0.5 & 0.0 \\
\hline 1500 & 0.2500 & 0.197 & 21.1 & 19.6 & 6.9 & 4.5 & 3.1 & 3.4 & 2.9 & -0.4 & -1.7 & -0.3 & 0.0 \\
\hline 1500 & 0.4000 & 0.145 & 27.4 & 24.3 & 10.6 & 7.8 & 4.1 & 7.3 & 6.0 & -1.7 & -3.2 & -2.1 & 0.0 \\
\hline 1500 & 0.6500 & 0.014 & 38.9 & 35.4 & 14.5 & 10.7 & 6.4 & 7.2 & 5.8 & -0.3 & -2.7 & -3.3 & 0.0 \\
\hline 2000 & 0.0320 & 0.796 & 11.9 & 11.1 & 3.9 & 0.9 & $\overline{0.9}$ & 2.0 & 0.3 & -1.3 & 1.5 & 0.0 & -0.5 \\
\hline 2000 & 0.0500 & 0.599 & 13.9 & 13.0 & 4.4 & 1.7 & 1.8 & 2.3 & 1.3 & -1.4 & 1.2 & 0.2 & 0.0 \\
\hline 2000 & 0.0800 & 0.582 & 13.0 & 12.3 & 4.0 & 1.5 & 0.4 & 1.5 & 1.3 & -0.8 & 0.3 & 0.1 & 0.0 \\
\hline 2000 & 0.1300 & 0.224 & 20.6 & 20.0 & 4.4 & 1.7 & 0.9 & 1.5 & -1.0 & -0.9 & -0.6 & -0.4 & 0.0 \\
\hline 2000 & 0.1800 & 0.249 & 22.7 & 21.9 & 5.7 & 3.5 & 1.2 & 2.6 & 2.2 & 0.8 & 0.9 & 0.8 & 0.0 \\
\hline 2000 & 0.2500 & 0.197 & 23.4 & 22.4 & 6.1 & 3.8 & 1.2 & 2.9 & 2.6 & -1.2 & -0.2 & -0.6 & 0.0 \\
\hline 2000 & 0.4000 & 0.108 & 29.5 & 27.7 & 9.2 & 6.6 & 3.1 & 4.2 & 3.2 & -0.7 & -2.0 & -1.8 & 0.0 \\
\hline 3000 & 0.0500 & 0.606 & 12.4 & 10.6 & 5.8 & 2.0 & 2.2 & 2.7 & 0.6 & 0.2 & 1.9 & 0.2 & -1.7 \\
\hline 3000 & 0.0800 & 0.556 & 11.8 & 10.9 & 4.4 & 2.0 & 1.6 & 0.9 & -0.3 & -0.2 & 0.8 & 0.1 & 0.0 \\
\hline 3000 & 0.1300 & 0.464 & 13.0 & 12.4 & 3.9 & 0.9 & 0.6 & 1.0 & -0.8 & -0.4 & -0.5 & 0.2 & 0.0 \\
\hline 3000 & 0.1800 & 0.347 & 16.1 & 15.3 & 4.6 & 2.3 & 0.1 & 2.2 & 1.9 & -1.0 & 0.3 & 0.2 & 0.0 \\
\hline 3000 & 0.2500 & 0.255 & 19.1 & 17.8 & 6.4 & 4.3 & 1.7 & 2.9 & 2.7 & 0.3 & -0.6 & 0.6 & 0.0 \\
\hline 3000 & 0.4000 & 0.128 & 25.5 & 23.0 & 9.7 & 7.4 & 3.4 & 5.0 & 3.7 & 0.2 & -2.8 & -1.8 & 0.0 \\
\hline 5000 & 0.0800 & 0.707 & 11.7 & 10.6 & 4.4 & 1.4 & 2.1 & 2.0 & 0.4 & -0.7 & 1.8 & 0.1 & -0.3 \\
\hline 5000 & 0.1300 & 0.536 & 14.2 & 13.1 & 5.2 & 2.9 & 1.6 & 1.1 & 0.3 & 0.5 & 0.9 & 0.1 & 0.0 \\
\hline 5000 & 0.1800 & 0.442 & 14.9 & 14.0 & 5.1 & 3.0 & 0.3 & 0.9 & -0.7 & -0.5 & 0.3 & -0.3 & 0.0 \\
\hline 5000 & 0.2500 & 0.361 & 20.3 & 17.4 & 9.6 & 8.0 & 2.2 & 4.3 & 3.7 & 1.5 & -1.7 & 0.1 & 0.0 \\
\hline 5000 & 0.4000 & 0.091 & 33.5 & 31.6 & 10.9 & 8.9 & 1.2 & 1.9 & 1.5 & 0.8 & 0.8 & -0.3 & 0.0 \\
\hline 5000 & 0.6500 & 0.010 & 45.1 & 41.0 & 17.7 & 15.7 & 5.0 & 6.5 & 5.7 & 1.3 & -2.6 & -1.2 & 0.0 \\
\hline 8000 & 0.1300 & 0.722 & 17.2 & 16.0 & 6.2 & 3.4 & 2.1 & 1.9 & 1.4 & -0.2 & 1.3 & 0.3 & -0.3 \\
\hline 8000 & 0.1800 & 0.386 & 21.2 & 20.4 & 5.3 & 1.5 & 1.7 & 2.3 & -1.0 & -1.6 & 1.4 & 0.1 & 0.0 \\
\hline 8000 & 0.2500 & 0.295 & 23.3 & 21.8 & 7.4 & 5.2 & 0.8 & 3.5 & 2.8 & 2.0 & -0.7 & -0.2 & 0.0 \\
\hline 8000 & 0.4000 & 0.197 & 32.4 & 27.7 & 16.7 & 14.9 & 1.0 & 2.1 & 1.3 & 1.5 & 0.7 & 0.3 & 0.0 \\
\hline 12000 & 0.1800 & 0.471 & 28.8 & 27.8 & 7.1 & 1.9 & 0.7 & 2.7 & 1.6 & -1.8 & -1.2 & -0.3 & -0.1 \\
\hline 12000 & 0.2500 & 0.298 & 30.2 & 28.9 & 8.2 & 5.2 & 2.3 & 2.7 & -0.7 & -1.2 & 2.3 & 0.3 & 0.0 \\
\hline 12000 & 0.4000 & 0.083 & 53.7 & 50.0 & 18.1 & 16.2 & 0.2 & 7.6 & 6.3 & 4.3 & -0.7 & 0.0 & 0.0 \\
\hline 20000 & 0.2500 & 0.349 & 52.2 & 51.1 & 10.6 & 2.5 & 0.5 & 2.2 & 0.7 & 1.8 & 0.7 & 0.0 & -0.6 \\
\hline 20000 & 0.4000 & 0.182 & 46.7 & 44.7 & 13.1 & 8.8 & 1.9 & 2.3 & 1.4 & -1.6 & 0.9 & 0.0 & 0.0 \\
\hline 20000 & 0.6500 & 0.014 & 79.8 & 70.7 & 34.4 & 31.7 & 2.7 & 13.4 & 9.9 & 8.9 & -1.0 & 0.0 & 0.0 \\
\hline 30000 & 0.4000 & 0.268 & 72.9 & 70.7 & 16.9 & 4.8 & 0.7 & 4.7 & -3.5 & -2.4 & -2.0 & 0.0 & 0.0 \\
\hline
\end{tabular}




\begin{tabular}{|c|c|c|c|c|c|c|c|c|c|c|c|}
\hline $\begin{array}{c}Q^{2} \\
\left(\mathrm{GeV}^{2}\right)\end{array}$ & $x$ & $\begin{array}{c}\mathrm{d}^{2} \sigma_{C C} / \mathrm{d} x \mathrm{~d} Q^{2} \\
\left(\mathrm{pb} / \mathrm{GeV}^{2}\right)\end{array}$ & $\begin{array}{l}\delta_{t o t} \\
(\%)\end{array}$ & $\begin{array}{r}\delta_{\text {sta }} \\
(\%)\end{array}$ & $\begin{array}{c}\delta_{\text {unc }} \\
(\%)\end{array}$ & $\begin{array}{l}\delta_{u n c}^{h} \\
(\%)\end{array}$ & $\begin{array}{c}\delta_{\text {cor }} \\
(\%)\end{array}$ & $\begin{array}{r}\delta_{\text {cor }}^{V^{+}} \\
(\%)\end{array}$ & $\begin{array}{r}\delta_{\text {cor }}^{h^{+}} \\
(\%)\end{array}$ & $\begin{array}{l}\delta_{\text {cor }}^{N^{+}} \\
\quad(\%)\end{array}$ & $\begin{array}{l}\delta_{\text {cor }}^{B^{+}} \\
(\%)\end{array}$ \\
\hline 300 & 0.013 & $0.458 \cdot 10^{0}$ & 57.6 & 55.4 & 7.4 & 2.2 & 13.9 & 12.1 & -1.6 & 1.0 & -6.5 \\
\hline 300 & 0.032 & $0.399 \cdot 10^{0}$ & 27.3 & 24.5 & 5.2 & 2.3 & 10.9 & 10.0 & -2.0 & 1.8 & -3.3 \\
\hline 300 & 0.080 & $0.690 \cdot 10^{-1}$ & 42.3 & 40.7 & 8.7 & 2.3 & 7.6 & 4.7 & -1.7 & 1.4 & -5.6 \\
\hline 500 & 0.013 & $0.433 \cdot 10^{0}$ & 39.9 & 37.6 & 5.9 & 2.8 & 12.0 & 11.6 & -1.7 & 0.5 & -2.3 \\
\hline 500 & 0.032 & $0.285 \cdot 10^{0}$ & 21.0 & 19.6 & 5.0 & 3.0 & 6.0 & 3.7 & -1.8 & 0.4 & -4.3 \\
\hline 500 & 0.080 & $0.790 \cdot 10^{-1}$ & 22.4 & 21.8 & 4.3 & 3.0 & 2.8 & 1.1 & -1.4 & 2.1 & -0.4 \\
\hline 500 & 0.130 & $0.551 \cdot 10^{-1}$ & 29.9 & 29.0 & 6.5 & 3.0 & 2.7 & 0.2 & -1.3 & 2.2 & -0.7 \\
\hline 1000 & 0.032 & $0.186 \cdot 10^{0}$ & 18.2 & 17.5 & 4.4 & 1.8 & 2.2 & 1.5 & -1.1 & 1.0 & -0.6 \\
\hline 1000 & 0.080 & $0.556 \cdot 10^{-1}$ & 18.4 & 17.9 & 4.0 & 1.8 & 1.6 & 0.5 & -1.0 & 1.2 & 0.0 \\
\hline 1000 & 0.130 & $0.310 \cdot 10^{-1}$ & 24.5 & 24.0 & 4.4 & 1.8 & 1.4 & 0.1 & -1.0 & 1.0 & 0.0 \\
\hline 1000 & 0.250 & $0.139 \cdot 10^{-1}$ & 39.1 & 37.6 & 10.5 & 1.7 & 1.4 & 0.0 & -0.2 & -1.4 & 0.0 \\
\hline 2000 & 0.032 & $0.132 \cdot 10^{0}$ & 16.2 & 15.5 & 4.3 & 2.0 & 2.4 & 2.0 & -1.3 & -0.3 & -0.1 \\
\hline 2000 & 0.080 & $0.571 \cdot 10^{-1}$ & 13.6 & 13.0 & 3.8 & 2.0 & 1.1 & 0.6 & -0.5 & 0.7 & -0.1 \\
\hline 2000 & 0.130 & $0.197 \cdot 10^{-1}$ & 21.7 & 21.2 & 4.4 & 2.0 & 1.1 & 0.1 & -0.8 & 0.7 & -0.2 \\
\hline 2000 & 0.250 & $0.855 \cdot 10^{-2}$ & 26.4 & 25.6 & 6.5 & 0.7 & 0.8 & 0.0 & -0.7 & -0.3 & 0.0 \\
\hline 3000 & 0.080 & $0.324 \cdot 10^{-1}$ & 14.8 & 14.0 & 4.6 & 0.7 & 1.4 & 0.7 & -1.2 & 0.3 & 0.0 \\
\hline 3000 & 0.130 & $0.250 \cdot 10^{-1}$ & 15.2 & 14.0 & 6.1 & 0.7 & 0.7 & 0.2 & 0.4 & 0.6 & 0.0 \\
\hline 3000 & 0.250 & $0.749 \cdot 10^{-2}$ & 20.1 & 18.9 & 6.8 & 0.8 & 1.5 & 0.0 & -0.7 & 1.3 & 0.0 \\
\hline 3000 & 0.400 & $0.251 \cdot 10^{-2}$ & 40.3 & 35.2 & 17.1 & 4.4 & 9.7 & 0.0 & 1.7 & -9.5 & 0.0 \\
\hline 5000 & 0.080 & $0.213 \cdot 10^{-1}$ & 19.2 & 17.9 & 6.5 & 4.4 & 1.6 & $\overline{1.0}$ & 0.5 & 1.1 & 0.0 \\
\hline 5000 & 0.130 & $0.108 \cdot 10^{-1}$ & 18.2 & 16.8 & 7.0 & 4.4 & 0.8 & 0.1 & 0.7 & 0.4 & 0.0 \\
\hline 5000 & 0.250 & $0.550 \cdot 10^{-2}$ & 16.9 & 16.3 & 4.3 & 0.7 & 1.0 & 0.0 & -0.1 & 1.0 & 0.0 \\
\hline 5000 & 0.400 & $0.123 \cdot 10^{-2}$ & 35.6 & 33.1 & 12.4 & 6.2 & 4.3 & 0.0 & 3.6 & -2.4 & 0.0 \\
\hline 8000 & 0.130 & $0.722 \cdot 10^{-2}$ & 21.1 & 18.9 & 8.9 & 6.2 & 2.6 & 0.7 & 2.1 & 1.3 & 0.0 \\
\hline 8000 & 0.250 & $0.342 \cdot 10^{-2}$ & 17.4 & 16.3 & 6.0 & 2.5 & 1.5 & 0.1 & 1.5 & 0.1 & 0.0 \\
\hline 8000 & 0.400 & $0.946 \cdot 10^{-3}$ & 30.4 & 28.6 & 9.4 & 6.7 & 4.1 & 0.0 & 4.1 & 0.4 & 0.0 \\
\hline 15000 & 0.250 & $0.139 \cdot 10^{-2}$ & 27.3 & 22.1 & 15.4 & 7.1 & 4.4 & 0.2 & 4.2 & 1.3 & 0.0 \\
\hline 15000 & 0.400 & $0.419 \cdot 10^{-3}$ & 29.5 & 27.5 & 9.3 & 7.9 & 5.2 & 0.0 & 5.1 & 1.0 & 0.0 \\
\hline
\end{tabular}

Table 9: The CC double differential cross section $\mathrm{d}^{2} \sigma_{C C} / \mathrm{d} x \mathrm{~d} Q^{2}$ with total error $\left(\delta_{\text {tot }}\right)$, statistical error $\left(\delta_{s t a}\right)$, uncorrelated systematic error $\left(\delta_{u n c}\right)$, and its contributions from the hadronic energy error $\left(\delta_{u n c}^{h}\right)$. The effect of the other uncorrelated errors is included in $\delta_{\text {unc. In addition the }}$ correlated systematic error $\left(\delta_{c o r}\right)$, and its contributions from a positive variation of one standard deviation of the error coming from the anti-photoproduction cut $\left(\delta_{c o r}^{V^{+}}\right)$, of the hadronic energy error $\left(\delta_{c o r}^{h^{+}}\right)$, of the noise contribution $\left(\delta_{c o r}^{N^{+}}\right)$and of the error due to background subtraction $\left(\delta_{c o r}^{B^{+}}\right)$. The normalisation uncertainty of $1.8 \%$ is not included in the errors. 


\begin{tabular}{|r|c|c|c|c|c|}
\hline $\begin{array}{r}Q^{2} \\
\left(\mathrm{GeV}^{2}\right)\end{array}$ & $x$ & $x \tilde{F}_{3}$ & $\delta_{\text {sta }}$ & $\delta_{\text {sys }}$ & $\delta_{\text {tot }}$ \\
\hline \hline 1500 & 0.020 & 0.0529 & 0.0416 & 0.0284 & 0.0503 \\
1500 & 0.032 & 0.0972 & 0.0433 & 0.0353 & 0.0558 \\
1500 & 0.050 & 0.1028 & 0.0597 & 0.0442 & 0.0743 \\
1500 & 0.080 & 0.0926 & 0.0840 & 0.0617 & 0.1042 \\
\hline 5000 & 0.080 & 0.1778 & 0.0360 & 0.0238 & 0.0431 \\
5000 & 0.130 & 0.2084 & 0.0506 & 0.0346 & 0.0613 \\
5000 & 0.180 & 0.1667 & 0.0619 & 0.0377 & 0.0725 \\
5000 & 0.250 & 0.1253 & 0.0787 & 0.0619 & 0.1002 \\
5000 & 0.400 & 0.0374 & 0.0823 & 0.0661 & 0.1055 \\
5000 & 0.650 & -0.0120 & 0.0285 & 0.0152 & 0.0323 \\
\hline 12000 & 0.180 & 0.1922 & 0.0851 & 0.0225 & 0.0880 \\
12000 & 0.250 & 0.1352 & 0.0687 & 0.0234 & 0.0726 \\
12000 & 0.400 & -0.0057 & 0.0645 & 0.0338 & 0.0728 \\
12000 & 0.650 & -0.0141 & 0.0281 & 0.0163 & 0.0325 \\
\hline
\end{tabular}

Table 10: The structure function $x \tilde{F}_{3}$ with statistical $\left(\delta_{\text {sta }}\right)$, systematic $\left(\delta_{\text {sta }}\right)$, and total $\left(\delta_{\text {tot }}\right)$ absolute errors. The luminosity uncertainties of the $e^{+} p$ and $e^{-} p$ data sets are included in the systematic error. 